\title{
miR-665 expression predicts poor survival and promotes tumor metastasis by targeting NR4A3 in breast cancer
}

\author{
Xin-Ge Zhao', Jing-Ye Hư ${ }^{2}$, Jun Tang ${ }^{3}$, Wei Yi ${ }^{4}$, Mei-Yin Zhang ${ }^{1}$, Rong Deng ${ }^{1}$, Shi-Juan Mai ${ }^{1}$, Nuo-Qing Weng ${ }^{1}$, \\ Rui-Qi Wang ${ }^{1}$, Ji Liu', Hui-Zhong Zhang', Jie-Hua He ${ }^{5}$ and Hui-Yun Wang ${ }^{1}$
}

\begin{abstract}
Cancer metastasis is the main cause of death in breast cancer (BC) patients. Therefore, prediction and treatment of metastasis is critical for enhancing the survival of BC patients. In this study, we aimed to identify biomarkers that can predict metastasis of $B C$ and elucidate the underlying mechanism of the functional involvement of such markers in metastasis. miRNA expression profile was analyzed using a custom microarray system in $422 \mathrm{BC}$ tissues. The relationship between the upregulated miR-665, metastasis and survival of $B C$ was analyzed and verified in another set of 161 BC samples. The biological function of miR-665 in BC carcinogenesis was explored with in vitro and in vivo methods. The target gene of miR-665 and its signaling cascade were also analyzed. There are 399 differentially expressed miRNAs between $B C$ and noncancerous tissues, of which miR-665 is the most upregulated miRNA in the BC tissues compared with non-tumor breast tissues $(P<0.001)$. The expression of miR-665 predicts metastasis and poor survival in 422 BC patients, which is verified in another 161 BC patients and 2323 BC cases from online databases. Ectopic miR-665 expression promotes epithelial-mesenchymal transition (EMT), proliferation, migration and invasion of BC cells, and increases tumor growth and metastasis of BC in mice. Bioinformatics, luciferase assay and other methods showed that nuclear receptor subfamily 4 group A member 3 (NR4A3) is a target of miR-665 in BC. Mechanistically, we demonstrated that miR-665 promotes EMT, invasion and metastasis of BC via inhibiting NR4A3 to activate MAPK/ERK kinase (MEK) signaling pathway. Our study demonstrates that miR-665 upregulation is associated with metastasis and poor survival in BC patients, and mechanistically, miR-665 enhances progression of BC via NR4A3/ MEK signaling pathway. This study provides a new potential prognostic biomarker and therapeutic target for $B C$ patients.
\end{abstract}

\section{Introduction}

Breast cancer $(\mathrm{BC})$ is the second most common cancer in the world and is the fifth leading cause of cancer death among women ${ }^{1}$. A recent report shows that $\mathrm{BC}$ ranked

\footnotetext{
Correspondence: Hui-Yun Wang (wanghyun@mail.sysu.edu.cn)

${ }^{1}$ State Key Laboratory of Oncology in South China, Collaborative Innovation

Center for Cancer Medicine, Sun Yat-Sen University Cancer Center, Guangzhou 510060, China

${ }^{2}$ Department of Basic Medicine, Guiyang College of Traditional Chinese Medicine, Guiyang 550002, China

Full list of author information is available at the end of the article.

These authors contributed equally: Xin-Ge Zhao, Jing-Ye Hu
}

Edited by R. Aqeilan first among young adults aged 20-39 years in terms of new cases and deaths in the world ${ }^{2}$. In 2017, approximately 252,710 new cases of female breast cancer are expected to be diagnosed in the United States and about 40,610 women will die from this disease ${ }^{3}$. Importantly, it is not the local tumor itself but the result of metastasis to the lymph nodes or other organs that leads to death. Therefore, prediction, prevention and treatment of $\mathrm{BC}$ metastasis are critically important to enhance survival of $\mathrm{BC}$ patients ${ }^{4,5}$. To this purpose, a better understanding of the molecular mechanisms underlying the metastasis of $\mathrm{BC}$ is desperately needed and will facilitate the

\section{(c) The Author(s) 2019}

(c) (i) Open Access This article is licensed under a Creative Commons Attribution 4.0 International License, which permits use, sharing, adaptation, distribution and reproduction in any medium or format, as long as you give appropriate credit to the original author(s) and the source, provide a link to the Creative Commons license, and indicate if changes were made. The images or other third party material in this article are included in the article's Creative Commons license, unless indicated otherwise in a credit line to the material. If material is not included in the article's Creative Commons license and your intended use is not permitted by statutory regulation or exceeds the permitted use, you will need to obtain permission directly from the copyright holder. To view a copy of this license, visit http://creativecommons.org/licenses/by/4.0/. 
identification of novel biomarkers for prediction and therapeutic targets for treatment of metastasis in $\mathrm{BC}$ patients.

MicroRNAs (miRNAs) are a class of noncoding RNAs of about 22 nucleotides in length that negatively regulate posttranscriptional gene expression by either repressing the targeted mRNA translation or degrading the mRNAs via partly binding to mRNA's $3^{\prime}$ untranslated region $(\mathrm{UTR})^{6}$. MiRNAs are now widely recognized to regulate diverse cellular processes in both normal and tumor cells. Studies also show that the abnormally expressed miRNAs are involved in breast tumorigenesis and metastasis by acting as a tumor suppressor or tumor activator ${ }^{7-10}$. However, the role and mechanism of miRNA in BC still have not been fully elucidated ${ }^{11-13}$.

To investigate the role of miRNA in BC development and progression, we examined miRNA expression profile in 422 $\mathrm{BC}$ tissues by using a custom microarray system and found that miR-665 was upregulated in $\mathrm{BC}$ tissues and associated with poor survival of BC patients. Previous research also reported upregulation of miR-665 in hepatocellular carcinoma, non-small cell lung cancer and intestinal gastric adenocarcinoma ${ }^{14-16}$ and downregulation in gastric signet ring cell carcinoma, osteosarcoma, and pancreatic cancer $^{16-18}$. The role of miR-665 in BC, however, has not been explored yet although one report mentioned miR665 downregulation in $48 \mathrm{BC}$ tissues $^{19}$.

In the present study, we demonstrate that miR-665 is significantly upregulated in $\mathrm{BC}$ and markedly correlated with tumor distant metastasis and poor prognosis of $\mathrm{BC}$. Furthermore, miR-665 acts as a potent oncomiR that promotes cell proliferation, invasion, and metastasis in vitro and in vivo by targeting NR4A3.

\section{Materials and methods Patients and samples}

In this study, 422 archived formalin fixed paraffinembedded (FFPE) BC samples and 31 FFPE noncancerous breast tissues (NBT) were obtained from the Department of Pathology, Sun Yat-Sen University Cancer Center (SYSUCC), Guangzhou, China. The 422 BC patients with breast cancer underwent curative mastectomy and were pathologically diagnosed as invasive ductal carcinoma (IDC) between June of 1999 and March of 2005. Another 161 FFPE BC samples from patients who received curative mastectomy and pathologically diagnosed as IDC between June of 2002 and December of 2006, were collected from the First Affiliated Hospital of Guangzhou Medical University (FAHGMU), Guangzhou, China. Tumor node metastasis (TNM) staging was performed according to the American Joint Committee on Cancer staging manual (sixth edition, 2002). The clinical characteristics of patients in the 2 cohorts are summarized in Table 1 . The median follow-up time was
94.3 months (range from 3 to 142) for 422 patients from SYSUCC and 75.2 months (range from 3 to 116) for 161 patients from FAHGMU. Overall survival (OS) was determined from the date of surgery to the date of death due to any cause or the last follow-up date; disease-free survival (DFS) from the date of surgery to the date of the first distant metastasis, relapse, death or the last follow-up date; distance metastasis-free survival (DMFS) from the date of surgery to the date of the first distant metastasis or death. This study was approved by the Ethical Committees of Sun Yat-Sen University Cancer Center and Affiliated Hospital of Guangzhou Medical University. The written informed consent was obtained from every patient in the two cohorts.

\section{Microarray assay}

The custom miRNA microarray employed in this study had been fabricated in house and hybridized with total RNAs as previously described ${ }^{20,21}$. Briefly, 1849 probes had been successfully designed based on all human mature miRNAs (1921) in the miRBase database (Release 18.0) according to the principle proposed by Wang et al. ${ }^{22}$. All probes (20-22 nt, $40 \mu \mathrm{M}$ final concentration) mixed with printing buffer were printed on cleaned slides, and the microarray was hybridized at $45^{\circ} \mathrm{C}$ for $16 \mathrm{~h}$ with $2 \mu \mathrm{g}$ total RNA labeled with Cy3 or Cy5. After washing three times, microarray was dried and scanned. After subtracting background, the microarray data were normalized with the quantile normalization method and log transformed.

\section{Cell culture and transfection}

A normal human breast epithelial cell line, MCF-10A and six human breast cancer cells T47D, MCF-7, MDAMB-231, MDA-MB-415, ZR-75-1, and ZR-75-30 cell lines were maintained at the State Key Laboratory of Oncology in South China. MCF-10A cells were cultured in a complete growth medium (CM-0525, Procell Life Science \& Technology Company, Wuhan, China). BC cells were maintained in Dulbecco's modified Eagle medium (DMEM containing $4.5 \mathrm{~g} / \mathrm{L}$ D-glucose) supplemented with $10 \%$ fetal bovine serum (FBS; Gibco, US) at $37^{\circ} \mathrm{C}$ in a humidified incubator with an atmosphere of $5 \% \mathrm{CO}_{2}$. The miR-665 mimics, miR-665 inhibitor, normal (scramble) control (NC) oligonucleotides, and the small interfering RNA (siRNA) targeting human NR4A3 mRNA were purchased from GenePharma (Shanghai, China). Cell transfection was performed using Lipofectamine ${ }^{\mathrm{Tm}} 3000$ transfection reagent (Invitrogen, USA) according to the manufacturer's instructions.

\section{Lentivirus production and transduction}

The recombinant lentiviral vectors containing miRNA665 , short hairpin RNA against human miRNA-665 or scrambled control oligonucleotides were purchased from 
Table 1 Clinicopathological characteristics of patients with $B C$ in the two cohorts

\begin{tabular}{|c|c|c|}
\hline Characteristics & $\begin{array}{l}\text { SYSUCC } \\
n(\%)\end{array}$ & $\begin{array}{l}\text { FAHGMU } \\
n(\%)\end{array}$ \\
\hline \multicolumn{3}{|l|}{ Age } \\
\hline$\geq 50$ & $169(40.0)$ & $84(52.2)$ \\
\hline$<50$ & $253(60.0)$ & $77(47.8)$ \\
\hline \multicolumn{3}{|l|}{ Menopause } \\
\hline Yes & $164(38.9)$ & $75(46.6)$ \\
\hline No & $258(61.1)$ & $86(53.4)$ \\
\hline \multicolumn{3}{|c|}{ Pathological Grade } \\
\hline I & $17(4.0)$ & $7(4.3)$ \\
\hline$\|$ & $345(81.8)$ & $134(83.2)$ \\
\hline III & $60(14.2)$ & $20(12.5)$ \\
\hline \multicolumn{3}{|l|}{ Estrogen receptor } \\
\hline Positive & $246(58.3)$ & $103(64.0)$ \\
\hline Negative & $176(41.7)$ & $58(36.0)$ \\
\hline \multicolumn{3}{|c|}{ Progesterone receptor } \\
\hline Positive & $234(55.5)$ & $101(62.7)$ \\
\hline Negative & $188(44.5)$ & $60(37.3)$ \\
\hline \multicolumn{3}{|l|}{ Her-2 } \\
\hline Positive & $120(28.4)$ & $104(64.6)$ \\
\hline Negative & $302(71.6)$ & $57(35.4)$ \\
\hline \multicolumn{3}{|l|}{ TNBC } \\
\hline Yes & $46(10.9)$ & $13(8.1)$ \\
\hline No & $376(89.1)$ & $148(91.9)$ \\
\hline \multicolumn{3}{|l|}{ T stage } \\
\hline $\mathrm{T} 1-2$ & $363(86.0)$ & $138(27.5)$ \\
\hline T3-4 & $59(14.0)$ & $23(28.0)$ \\
\hline \multicolumn{3}{|l|}{ N stage } \\
\hline No-1 & $301(71.3)$ & $131(81.4)$ \\
\hline N2-3 & $121(28.7)$ & $30(18.6)$ \\
\hline \multicolumn{3}{|l|}{ Distant metastasis } \\
\hline Yes & $50(11.8)$ & NA \\
\hline No & $372(88.2)$ & NA \\
\hline \multicolumn{3}{|l|}{ TNM stage } \\
\hline I & $71(73.2)$ & $26(26.8)$ \\
\hline$\|$ & $214(69.7)$ & $93(30.3)$ \\
\hline III & $137(76.5)$ & $42(24.5)$ \\
\hline
\end{tabular}

GenePharma (Shanghai, China). MCF-7, MDA-MB-231 and ZR-75-30 cells were infected with recombinant lentivirus plus $5 \mu \mathrm{g} / \mathrm{ml}$ polybrene (Sigma, St. Louis, MO,
USA) according to the manufacturer's instructions. Stable transfected cells were selected by using puromycin $(1 \mu \mathrm{g} /$ $\mathrm{ml}$ ) for 2 weeks. Green flourescent protein-positive cells were collected and used for subsequent assays.

\section{Quantitative real-time PCR (qRT-PCR)}

Paraffin-embedded breast cancer tissues and noncancerous breast tissues were cut into $8-10 \mu \mathrm{m}$ sections, which were then scraped off the slides and collected into a tube with a modified needle. The paraffin-embedded debris tissues were deparaffinized thrice with xylene. Total RNA was extracted from the debris tissues using the phenol/chloroform extraction method as described previously ${ }^{20,23}$ and extracted from cultured cells or fresh tissues using the TRIzol (Invitrogen, USA) according to the manufacturer's instruction. For the gene-expression assay, $1 \mu \mathrm{g}$ of total RNA was used to synthesize cDNA in a $20 \mu \mathrm{L}$ total volume using a PrimeScript RT reagent kit (Promega, Madison, WI, USA) and $0.4 \mu \mathrm{L}$ of cDNA product was used for quantitative PCR with the Platinum SYBR Green qPCR SuperMix-UDG kit. For miRNA quantitative real-time polymerase chain reaction (qRTPCR), 10 ng of total RNA isolated from FFPE samples were analyzed by the TaqMan method; or $1 \mu \mathrm{g}$ of total RNA extracted from cell lines and miR-665 primers or U6 primers were used to reverse transcribe with PrimeScript RT reagent kit in $20 \mu \mathrm{L}$ of total volume, and $0.4 \mu \mathrm{L}$ of the RT products was applied to quantitative PCR with BulgeLoop $^{\text {Tw }}$ miRNA qRT-PCR reagent kit (RIBOBIO, Guangzhou, China). The PCR reaction cycle was as follows: $50^{\circ} \mathrm{C}$ for $2 \mathrm{~min}$ and then $95^{\circ} \mathrm{C}$ for $10 \mathrm{~min}$ followed by 35 or 40 cycles of $95^{\circ} \mathrm{C}$ for $15 \mathrm{~s}$ and $60^{\circ} \mathrm{C}$ for $60 \mathrm{~s}$. Each sample was analyzed in triplicate. Glyceraldehyde-3-phosphate dehydrogenase (GAPDH) was used as an internal control to calculate the relative gene expression and the universal small nuclear RNA (snRNA) U6 was used as an endogenous control to calculate the relative miR-665 expression. miRNA or gene expression level measured by qRT-PCR was presented as $2^{-\Delta \Delta C T}$. The primers for miR-665, control U6, and other genes are listed in Table S1.

\section{Western blot analysis}

Cultured cells were harvested and lysed in RIPA buffer containing phenylmethylsulfonyl fluoride (PMSF) (100:1), protease inhibitor (100:1) and phosphatase inhibitor (1000:1) for $30 \mathrm{~min}$ on ice. Then the lysed solution was centrifuged at $12,000 \mathrm{~g}, 4{ }^{\circ} \mathrm{C}$ for $15 \mathrm{~min}$. Protein in the supernatant was collected and its concentration was measured using a Bicinchoninic Acid Assay kit (BOSTER, Pleasanton, CA). Approximately $10-20 \mu \mathrm{g}$ of protein were denatured at $100^{\circ} \mathrm{C}$ for $10 \mathrm{~min}$ with DualColor Protein Loading Buffer (Life, USA). The denatured proteins were separated in $10 \%$ sodium dodecyl sulfate-polyacrylamide gel electrophoresis. After electrophoresis, proteins were 
transferred onto polyvinylidene difluoride membranes (GE Healthcare Life Sciences, UK) using a Bio-Rad MiniTrans-Blot apparatus at $100 \mathrm{~V}$ for $2 \mathrm{~h}$ as suggested by the manufacturer. The membranes were blocked in 5\% nonfat milk for $2 \mathrm{~h}$ followed by incubation with primary monoclonal antibodies overnight at $4{ }^{\circ} \mathrm{C}$ with soft shaking. After 3 washes with Tris-buffered saline and Tween 20, the membranes were subsequently incubated with the corresponding secondary antibody coupled to horseradish peroxidase (HRP) at room temperature for $2 \mathrm{~h}$ and developed in electrochemiluminescence detection reagent (Advansta, Wuhan, China). The primary antibodies against human E-cadherin, N-cadherin, Vimentin, $\beta$-catenin, Slug, extracellular signal-regulated kinase (ERK), p-ERK, and GAPDH were purchased from Cell Signal Technology (Boston, USA). NR4A3 antibody was purchased from Santa Cruz Biotechnology (Dallas, USA).

\section{Immunofluorescence assay}

Immunofluorescence assay was performed $48 \mathrm{~h}$ after MCF-7 cells were transfected with miR-665 mimics or NC. The BC cells first were fixed with $4 \%$ paraformaldehyde for $15 \mathrm{~min}$ and washed 3 times with PBS, and then permeabilized with $0.1 \%$ Triton X-100 for 10 min. After washing 3 times with PBS, the cells were blocked with $10 \% \mathrm{FBS}$ for $1 \mathrm{~h}$. Then the cells were incubated with antibodies against E-cadherin, Vimentin, and $\beta$-catenin (Cell Signaling Technology) at $4{ }^{\circ} \mathrm{C}$ overnight, respectively. The second antibody (goat anti-rabbit IgG) conjugated with Alexa Fluor 555 (Cell Signaling Technology, USA) was added and incubated at $37^{\circ} \mathrm{C}$ for $1 \mathrm{~h}$. After three washes with PBS, nuclei was co-stained with DAPI (Beyotime, Shanghai, China) for $15 \mathrm{~min}$. Finally, cell images were captured using a laser scanning confocal microscopy (Olympus, Japan).

\section{Cell proliferation assay}

Cell proliferation assays were performed using a Cell counting kit-8 (CCK-8, Dojindo Laboratory, Kyushu, Japan) according to the manufacturer's instruction. Briefly, cells were seeded in 96-well plates at $2.0 \times 10^{3}$ cells/well and cultured for 1-7 days. At the indicated times, $10 \mu \mathrm{L}$ of CCK-8 solution was added to each well and incubated for additional $2 \mathrm{~h}$ at $37{ }^{\circ} \mathrm{C}$. Then the absorbance of the mixed solutions was measured at $450 \mathrm{~nm}$ with a SpectraMax M5 Multi-Mode Microplate Reader (Molecular Devices LLC, Sunnyvale, CA, USA). A calibration curve was prepared using absorbance obtained from wells that contained known numbers of viable cells.

\section{Colony formation assays}

Cells were seeded in 6-well plates (500 cells/well) and incubated for 14 days. Cells were fixed with methanol for
$10 \mathrm{~min}$ and stained with $0.1 \%$ crystal violet (Weijia Biology Science and Technology Co., Guangzhou, China) for $30 \mathrm{~min}$. Colonies containing more than 50 cells were counted under a microscope.

\section{Cell cycle analysis}

Cell cycle distribution was examined by flow cytometry using a cell cycle assay kit (BD Biosciences, San Jose, CA, USA) according to the manufacturer's protocol. After propidium iodide (PI) staining, the cellular DNA content was used to determine cell cycle profile (G0/G1, G2/M and $S$ phases), and the distribution of cell cycle was analyzed using Cell-FIT software (BD Biosciences, San Jose, CA, USA).

\section{Cell apoptosis analysis}

Cells were harvested and washed with ice-cold PBS thrice. Then, the cells were stained with Annexin Vfluorescein isothiocyanate and PI according to the manufacturer's instruction (BD Biosciences, San Jose, CA, USA) and examined with flow cytometry. The apoptotic cells were determined using Cell-FIT software (BD Biosciences, San Jose, CA, USA).

\section{Cell migration, wound healing, and invasion assays}

For migration assays, cells $\left(5 \times 10^{5}\right)$ in $200 \mu \mathrm{L}$ of serumfree DMEM medium were seeded in the upper chamber (BD Biosciences) with $8-\mu \mathrm{m}$ pore membrane of an insert and placed in the 24-well culture plate. DMEM medium $(700 \mu \mathrm{L})$ containing 10\% FBS was added to the lower chamber as a chemoattractant. After $16 \mathrm{~h}$, the nonmigratory cells remaining in the upper chamber were gently removed with a cotton swab. The migrated cells located on the lower side of the insert were fixed with methanol for $10 \mathrm{~min}$ and stained with crystal violet (Weijia Biology Science and Technology Co., Guangzhou, China) for $30 \mathrm{~min}$ at room temperature. Five random fields per well were observed, and cells were counted under the microscope. In addition, cell migration was also assessed with a scratch wound healing assay. The cells were seeded in a six-well plate. When the cells reached subconfluence, a scratch wound was generated with a sterile micropipette tip, and the 10\% FBS medium was replaced with serum-free DMEM. The scratch width was observed every $24 \mathrm{~h}$ and photographed under a microscope and the results were presented as percent scratch closure.

For invasion assays, cells $\left(5 \times 10^{5}\right)$ in $200 \mu \mathrm{L}$ of serumfree DMEM medium were seeded in the chamber (BD Biosciences) with matrigel-coated 8 - $\mu \mathrm{m}$ pore membrane of an insert and the insert was positioned in a 24-well culture plate. The subsequent steps were carried out as the same as the migration assay protocol. 


\section{Luciferase reporter assay}

To confirm the direct regulating relationship between miR-665 and NR4A3, the putative miR-665 recognition element wild type and mutant sequences in the $3^{\prime}$ UTR of NR4A3 gene was cloned into a SV40-firefly luciferase reporter vector (Applied Genechem., Shanghai, China) for NR4A3. For luciferase reporter assay, the constructed report vectors containing wild-type fragment or mutanttype fragment together with renilla vector and miR-665 mimics or miR-NC were co-transfect into MCF-7 or MDA-MB-231 cells using Lipofectamine ${ }^{\mathrm{TM}} 3000$ reagent. The transfected cells were incubated for $48 \mathrm{~h}$ and prepared for measuring the luminescence signals according to instruction of Dual-Luciferase Reporter Assay System kit (Promega, Madison, WI, USA). The firefly luciferase activity was normalized based on Renilla luciferase activity.

To demonstrate that overexpressed miR-665 induces transcriptional activation of $\beta$-catenin, the promoter of $\beta$-catenin was cloned into the Gaussia Luciferase (Gluc) reporter vector (Genecopoeia, Rockville, MD, USA), a dual-reporter system consisting of secreted GLuc and Alkaline Phosphatase (SEAP). Then the dual-reporter system and miR-665 NC or miR-665 mimics, were cotransfected into MCF-7 or MDA-MB-231 cells with Lipofectamine $^{\text {TM }} 3000$ reagent. Luciferase activity was detected with Secrete-Pair ${ }^{\mathrm{TM}}$ Dual Luminescence Assay Kit (Genecopoeia) according to the manufacturer's instructions. The SEAP signal was used as a transfection efficiency internal control and luminescence intensities were detected by Tecan Spark ${ }^{\mathrm{TM}} 10 \mathrm{M}$.

\section{Tumor formation and metastasis assays in nude mice}

Tumor formation assay: 16 female BALB/c nude mice (4-5 week old) were injected subcutaneously with $1 \times 10^{7}$ LV-miR-665-ZR-75-30 cells or LV-miR-control-ZR-75-30 cells separately. Then the subcutaneous xenografts were observed and measured every 3 days. After 24 days, the mice were euthanized and the formed tumors were resected and weighted. Tumor size was measured using calipers, and tumor volumes were calculated according to the formula: $V=a^{*} b^{2} / 2$, where $a$ and $b$ are the largest and shortest diameter in $\mathrm{mm}$, respectively. Then, the resected tumors were fixed in $10 \%$ formalin and embedded in paraffin blocks for pathological examination.

Metastasis assays: 16 male BALB/c nude mice (3-4 week old) were randomized into two groups. Then, $100 \mu \mathrm{L}$ of cell suspension containing $1 \times 10^{7} \mathrm{LV}$-miR-665-ZR-75-30 cells or LV-miR-control-ZR-75-30 cells was injected intravenously through the tail vein into each mouse. The experiment was terminated after 8 weeks, the mice were euthanized and the lungs were removed and fixed with $10 \%$ formalin. Subsequently, consecutive tissue sections were made from each block of the lung. The sections were stained with hematoxylin-eosin staining (H\&E).
The animals were housed under standard conditions and were supplied with food and water ad libitum according to the institutional guidelines for animal care. The experiments were performed in accordance with the guidelines of the Laboratory Animal Ethics Committee of Sun Yat-Sen University.

\section{IHC staining}

IHC analysis and qualification of NR4A3 expression were performed using a standard streptavidin-biotin-peroxidase complex method $^{24}$. Briefly, fresh tissue specimens were fixed in $10 \%$ formaldehyde and routinely processed for paraffin embedding. Then these blocks were cut into $4 \mu \mathrm{m}$ thick sections. Hydrogen peroxide (3\%) in methanol was used to block endogenous peroxidase activity. After antigen retrieval with microwave heating, the sections were incubated with rabbit polyclonal NR4A3 antibody (1:100, Signalway Antibody LLC, Maryland, USA) overnight at $4{ }^{\circ} \mathrm{C}$. Then, the sections were incubated with HRP-conjugated anti-rabbit IgG secondary antibody (KeyGEN, Guangzhou, China) for $30 \mathrm{~min}$ at room temperature, followed by development using 3, 5-diaminobenzidine (DAB, KeyGEN, Guangzhou, China) substrate and counterstaining with hematoxylin for the nuclei.

\section{Gene set enrichment analysis}

The information of $\mathrm{BC}$ samples with highest or lowest levels of miR-665 ( $n=10$, respectively) in TCGA database were downloaded and the fold change between the two groups was more than eight times. Gene set enrichment analysis was carried out using nonparametric scores with all default settings.

\section{Online bioinformatics analysis}

In the Kaplan-Meier plotter database (http://kmplot. com/analysis/), we analyzed the relationship between gene expression (miR-665 and NR4A3) and BC patients' survival including OS, relapse-free survival (RFS) and DMFS with Kaplan-Meier estimator. Putative miR-665 target genes were predicted using the miRNA target prediction algorithm TargetScan (http://www.targetscan. org/) and miRDB database (http://www.mirdb.org/).

\section{Statistical analysis}

Chi squared $\left(\chi^{2}\right)$ test was used for analyzing the relationships between miR-665 expression and clinicopathological features of patients with breast cancer. Survival curves were plotted using the Kaplan-Meier method and compared using a log-rank test. Student's $t$ test was used to test the differences of clinical features between two groups. All of statistic analyses and plots were performed in SPSS version 17.0 software (SPSS, Inc., Chicago, IL, USA) and GraphPad Prism 5 software (GraphPad Software, San Diego, CA, USA). ImageJ 
software was used to analyze cell migration, invasion and protein expression.

\section{Results \\ miR-665 expression is significantly elevated in BC tissues and cell lines}

In order to identify the miRNA expression profile in $\mathrm{BC}$, total RNAs from 422 breast cancer specimens and 31 noncancerous breast tissues obtained from SYSUCC were detected using our custom miRNA microarray containing 1849 probes $^{20}$. The result showed that 399 of the probed 1849 miRNAs were differentially expressed between BC and noncancerous tissues, in which 193 miRNAs were upregulated and 206 downregulated in BC tissues (detailed results will be published in another paper). Among the upregulated miRNAs, miR-665 was significantly upregulated in the BC tissues compared with non-tumor tissues examined by our microarray system $(P$ $<0.001$ ) (Fig. 1a). Next, we investigated the expression levels of miR-665 in several BC cell lines with real-time RT-PCR analysis, and found a markedly higher level of miR-665 in MCF-7, MDA-MB-415, and ZR-75-30 cell lines, and lower levels in T47D, MDA-MB-231, and ZR75-1 cell lines when the data were normalized to the normal breast epithelial cell line, MCF-10A (Fig. 1b), indicating that miR-665 is overexpressed in 50\% (3/6) of our examined BC cell lines.

\section{Overexpression of miR-665 is associated with poor survival and tumor metastasis in $\mathrm{BC}$ patients}

In order to evaluate the clinical significance of the overexpressed miR-665, we first analyzed the relationship between clinical characteristics and miR-665 expression in SYSUCC cohort. Patients were divided into high- or low-level group by the median value of miR-665 expression in BC tissues. The result is presented in Table 2, which shows that high expression of miR-665 is significantly associated with higher $\mathrm{T}$ stage $(P=0.035), \mathrm{N}$ stage $(P=0.041)$, TNM stage $(P<0.001)$, and postoperative metastasis $(P=0.007)$. Next, we examined the association of miR-665 expression with the survival of BC patients. Patients with high miR-665 expression in their tumors had much poorer OS, DFS, and DMFS than those with low miR-665 expression (Fig. 1c-e). Cox regression analysis of the microarray data indicated that miR-665 expression and TNM staging were independent prognostic indicators for OS and DFS of BC patients (Table 3). All these results suggest that miR-665 plays an important role in $\mathrm{BC}$ progression and metastasis.

To confirm the clinical significance of miR-665 overexpression, we detected miR-665 expression with qRTPCR in FAHGMU cohort (161 BC samples) and analyzed its correlation with survival. Survival analysis indicated that high miR-665 expression is related with poor OS and
DFS (Fig. 1f, g), and Cox regression analysis showed that miR-665 is an independent prognostic factor for OS and DFS (Table 4), which is consistent with the results obtained from SYSUCC cohort. These results demonstrate that miR-665 high-expression not only was significantly correlated with poor survival but also with elevated risk of metastasis.

Next, we wondered if the overexpressed miR-665 had the same clinical significance in $\mathrm{BC}$ patients from different geographical areas of the world. To this purpose, we extracted the miR-665 data from Kapla-Meier plotter database (http://kmplot.com/analysis/) and assessed its relationship with the survival of $\mathrm{BC}$ patients. In this database, KM plot results obtained from $1061 \mathrm{BC}$ patients in TCGA dataset and 1262 BC patients in METABRIC dataset indicated that patients with a high level of miR665 had significantly poor OS than those with a low level (Fig. $1 \mathrm{~h}$ and Fig. S1A), which suggested that overexpressed miR-665 is ubiquitously correlated with poor survival of $\mathrm{BC}$ patients from different parts of the world. Furthermore, we found that miR-665 was only associated with poor survival in $\mathrm{BC}$ patients with estrogen receptor (ER)-positive tumor but not in patients with ER-negative tumor in both TCGA and METABRIC databases (Fig. $\mathrm{S} 1 \mathrm{~B}-\mathrm{E})$. Altogether, these results indicate that miR-665 is an onco-miRNA that mainly promotes the progression and metastasis of ER-positive BC.

miR-665 promotes BC cell proliferation and tumor growth

To substantiate the oncogenic role of miR-665 in BC, we constructed BC cells stably overexpressing or downregulating miR- 665 by lentivirus transduction with miR665 mimics or inhibitor in MCF-7, MDA-MB-231, and ZR-75-30 BC cell lines and analyzed cell proliferation by CCK-8 assay. Quantitative RT-PCR showed that miR-665 is overexpressed in cells stably transducted with miR-665 mimics and less-expressed in cells stably transducted with inhibitor when compared with their corresponding control cells (Fig. S2A-C). CCK-8 assay exhibited that the upregulation of miR-665 markedly promoted proliferation of MCF-7, MDA-MB-231, and ZR-75-30 cells, whereas the downregulation of miR-665 inhibited cell growth of these cell lines (Fig. 2a, b and Fig. S2D-G). Similarly, upregulation of miR-665 enhanced colony formation of $\mathrm{BC}$ cell, whereas downregulation of miR-665 reduced oncogenic colony formation in MCF-7 cells compared with the corresponding control cells (Fig. 2c, d). These results suggested that miR-665 could promote cell proliferation and drive oncogenic growth in BC cells. To investigate how miR-665 enhance BC cell proliferation or growth, we examined the effects of miR-665 on cell cycle and apoptosis by flow cytometry. The results revealed that the expressed miR-665 accelerated cell cycle progression by increasing the number of cells in $S$ phase, whereas the 

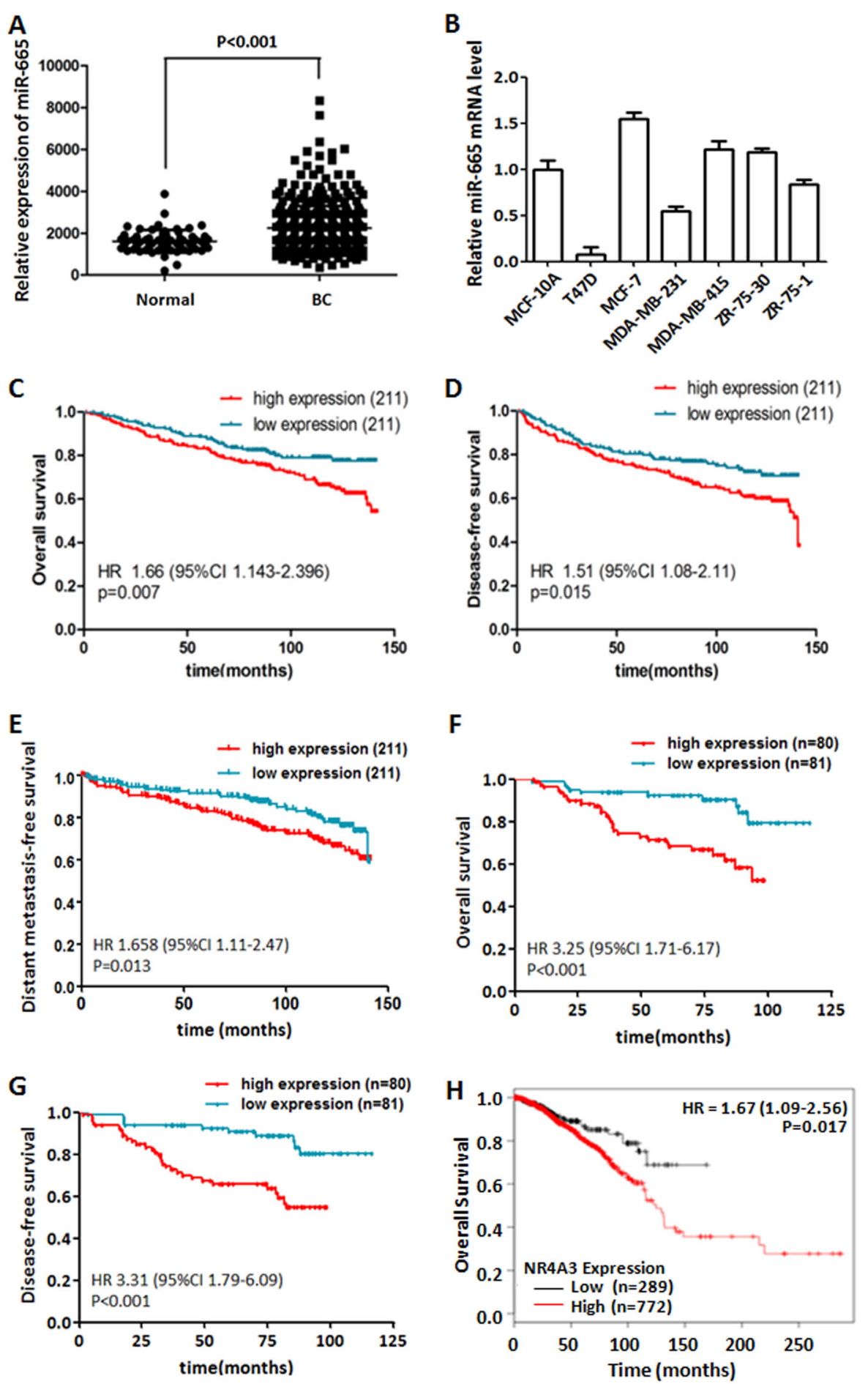

Fig. 1 miR-665 is significantly overexpressed in breast cancer $(B C)$ tissues and cell lines and associated with poor survivals of $B C$ patients. a The relative mRNA expression of miR-665 detected by microarray in $422 \mathrm{BC}$ tissues was significantly higher than that in 31 adjacent nontumor breast tissues ( $P<0.001$, independent Student's $t$ test). $\mathbf{b}$ The relative expression levels of miR-665 measured by qRT-PCR in MCF-7, MDA-MB-415, ZR75-30, T47D, MDA-MB-231, ZR-75-1 BC cell lines, and an immortalized breast epithelial cell line, MCF-10A. Relative miR-665 levels were determined by normalizing to U6 messenger RNA (mRNA) levels. c-e Patients with high level of miR-665 had a remarkably worse OS (c), DFS (d), and DMFS (e) than those with low level of miR-665 in SYSUCC cohort (422 cases), which was analyzed by Kaplan-Meier curve (log-rank test). f, $\mathbf{g}$ Patients with miR-665 high-expression also had a notably poorer OS (f) and DFS (g) than those with miR-665 low-expression in FAHGMU cohort (161 patients). $\mathbf{h}$ That patients with high miR-665 expression had a poor overall survival was confirmed in 1061 BC patients obtained from Kaplan-Meier plotter database 
Table 2 Association of miR-665 expression with clinicopathological characteristics in 422 BC patients

\begin{tabular}{|c|c|c|c|c|}
\hline \multirow[t]{2}{*}{ Characteristics } & \multirow[t]{2}{*}{$n$} & \multicolumn{2}{|c|}{ miR-665 expression level } & $P$ Value \\
\hline & & $\begin{array}{l}\text { Low level } \\
n(\%)\end{array}$ & $\begin{array}{l}\text { High level } \\
n(\%)\end{array}$ & \\
\hline
\end{tabular}

\begin{tabular}{|c|c|c|c|c|}
\hline \multicolumn{5}{|l|}{ Age } \\
\hline$\geq 50$ & 169 & $82(38.9)$ & $87(41.2)$ & 0.619 \\
\hline$<50$ & 253 & $129(61.1)$ & $124(58.8)$ & \\
\hline \multicolumn{5}{|l|}{ Menopause } \\
\hline Yes & 164 & $79(37.4)$ & $85(40.3)$ & 0.549 \\
\hline No & 258 & $132(62.6)$ & $126(59.7)$ & \\
\hline \multicolumn{5}{|c|}{ Pathological Grade } \\
\hline I & 17 & $6(2.8)$ & $11(5.2)$ & 0.419 \\
\hline$\|$ & 345 & $173(82.0)$ & $172(81.5)$ & \\
\hline III & 60 & $32(15.2)$ & $28(13.3)$ & \\
\hline \multicolumn{5}{|c|}{ Estrogen receptor } \\
\hline Positive & 246 & $124(58.8)$ & $122(57.8)$ & 0.843 \\
\hline Negative & 176 & $87(41.2)$ & $89(42.2)$ & \\
\hline \multicolumn{5}{|c|}{ Progesterone receptor } \\
\hline Positive & 234 & $118(55.9)$ & $116(55.0)$ & 0.845 \\
\hline Negative & 188 & $93(44.1)$ & $95(45.0)$ & \\
\hline \multicolumn{5}{|l|}{ Her-2 } \\
\hline Positive & 120 & $59(28.0)$ & $61(28.9)$ & 0.828 \\
\hline Negative & 302 & $152(72.0)$ & $150(71.1)$ & \\
\hline \multicolumn{5}{|l|}{ TNBC } \\
\hline Yes & 46 & $27(12.8)$ & $19(9.0)$ & 0.211 \\
\hline No & 376 & $184(87.2)$ & $192(91.0)$ & \\
\hline \multicolumn{5}{|l|}{ T stage } \\
\hline $\mathrm{T} 1-2$ & 363 & $189(52.1)$ & $174(47.9)$ & 0.035 \\
\hline T3-4 & 59 & $22(37.3)$ & $37(62.7)$ & \\
\hline \multicolumn{5}{|l|}{ N stage } \\
\hline No-1 & 301 & $141(46.8)$ & $160(53.2)$ & 0.041 \\
\hline N2-3 & 121 & $70(57.9)$ & $51(42.1)$ & \\
\hline \multicolumn{5}{|c|}{ Distant metastasis } \\
\hline No & 372 & $195(92.4)$ & 177 (83.9) & 0.007 \\
\hline Yes & 50 & $16(7.6)$ & $34(16.1)$ & \\
\hline \multicolumn{5}{|l|}{ TNM stage } \\
\hline I & 71 & $32(66.7)$ & $16(33.3)$ & $<0.001$ \\
\hline$\|$ & 214 & 79 (45.7) & $94(54.3)$ & \\
\hline III & 137 & $63(60.6)$ & $41(39.4)$ & \\
\hline
\end{tabular}

The bold values are less than 0.05 , which have statistic significance downregulation of miR-665 markedly arrested cell cycle progression by raising the number of cells in G1 phase when compared with the corresponding control cells (Fig. $2 \mathrm{e})$. In addition, flow cytometry analysis also revealed that ectopic expression of miR-665 inhibited cell apoptosis while the blockage of miR-665 expression increased cell apoptosis significantly (Fig. S2H, I). These in vitro results demonstrated that miR-665 promoted cell proliferation by accelerating G1/S phase transition and inhibiting apoptosis.

To further confirm the in vitro oncogenic role of miR665 , we subcutaneously injected miR-665 stably overexpressing cells (LV-miR-665-ZR-75-30 cells) and control cells into nude mice, respectively, to observe the effect of miR-665 on tumor growth in vivo. At 24 day after inoculation of tumor cells, the mice were euthanized and tumors were removed and measured. The results indicated that the volume and weight of tumors formed from LV-miR-665-ZR-75-30 cells $(n=8)$ were significantly higher than those formed from control cells $(n=8)$ $(P<0.05$, Fig. $2 \mathrm{f}-\mathrm{h})$, which demonstrated that miR-665 could also promote tumor growth in vivo.

\section{miR-665 is required for cell migration, invasion, lung metastasis and EMT in BC}

The result of the clinical analysis above shows that miR665 expression is associated with distant metastasis of BC. Therefore, we want to examine whether miR-665 can advance $\mathrm{BC}$ metastasis in vitro and in vivo. To this end, we first examined the effect of miR-665 on migration and invasion of $\mathrm{BC}$ cells with transwell assay. The number of migrating or invading MDA-MB-231 cells stably expressing miR-665 was much larger than that of the control MDA-MB-231 cells, whereas the number of migrated or invaded MDA-MB-231 cells stably expressing miR-665 inhibitor was much less than that of control cells (Fig. 3a, b), which suggested that miR-665 could enhance the metastatic potential of $\mathrm{BC}$ in vitro.

Next, we want to test if miR-665 increases the metastatic capability of BC cells in vivo. The LV-miR-665-ZR75-30 cells stably expressing miR-665 and the control cells were separately injected into nude mice through the tail vein. At 52 day after injection of $\mathrm{BC}$ cells, the remaining alive mice were euthanized. After removal, the lungs were fixed with $10 \%$ formalin and embedded in paraffin. Under a microscope, the metastatic lesions in the lung sections were calculated. The result showed that lung metastatic lesions in mice $(n=8)$ injected with miR-665expressing cells were significantly more than those in mice $(n=9)$ with control cells $(P<0.01$, Fig. $3 g$, h). Furthermore, 4 mice injected with miR-665-expressing cells died as a consequence of metastatic lesions in the lung within 52 days whereas none of the mice with control cells died in the same period (Fig. 3i), indicating that miR-665 
Table 3 Cox regression analysis of characteristics associated with survival of BC patients in SYSUCC cohort

\begin{tabular}{|c|c|c|c|c|}
\hline \multirow[t]{2}{*}{ Clinical characteristics } & \multicolumn{2}{|c|}{ Univariate Cox regression analysis } & \multicolumn{2}{|c|}{ Multivariate Cox regression analysis } \\
\hline & HR $(95 \% \mathrm{Cl})$ & $P$ Value & HR $(95 \% \mathrm{Cl})$ & $P$ Value \\
\hline \multicolumn{5}{|l|}{ Overall survival } \\
\hline miR-665 (high vs. low level) & $1.818(1.225-2.696)$ & 0.003 & $1.837(1.240-2.719)$ & 0.002 \\
\hline Age $(\geq 50$ vs. $<50)$ & $0.998(0.957-1.041)$ & 0.924 & & \\
\hline Menopause (yes vs. no) & $0.779(0.310-1.958)$ & 0.595 & & \\
\hline Pathological Grade (III vs. I and II) & $1.079(0.501-2.323)$ & 0.845 & & \\
\hline Her-2 (positive vs. negative) & $1.084(0.699-1.681)$ & 0.717 & & \\
\hline PR (positive vs. negative) & $0.708(0.451-1.111)$ & 0.133 & & \\
\hline ER (positive vs. negative) & $0.703(0.456-1.082)$ & 0.109 & & \\
\hline TNBC (positive vs. negative) & $0.968(0.531-1.765)$ & 0.916 & & \\
\hline TNM stage (III vs. II vs. I) & $2.287(1.660-3.151)$ & $<0.001$ & $2.392(1.240-2.719)$ & $<0.001$ \\
\hline \multicolumn{5}{|l|}{ Disease-free survival } \\
\hline miR-665 (high vs. low level) & $1.618(1.137-2.303)$ & 0.008 & $1.629(1.147-2.313)$ & 0.006 \\
\hline Age ( $\geq 50$ vs. $<50)$ & $0.916(0.962-1.035)$ & 0.998 & & \\
\hline Menopause (yes vs. no) & $0.701(0.316-1.555)$ & 0.383 & & \\
\hline Pathological Grade (III vs. I and II) & $1.215(0.629-2.346)$ & 0.562 & & \\
\hline Her-2 (positive vs. negative) & $1.068(0.723-1.578)$ & 0.74 & & \\
\hline PR (positive vs. negative) & $0.729(0.481-1.103)$ & 0.134 & & \\
\hline ER (positive vs. negative) & $0.697(0.483-1.004)$ & 0.053 & & \\
\hline TNBC (positive vs. negative) & $1.331(0.877-2.020)$ & 0.179 & & \\
\hline TNM stage (III vs. II vs. I) & $2.098(1.576-2.793)$ & $<0.001$ & $2.116(1.598-2.803)$ & $<0.001$ \\
\hline
\end{tabular}

The bold values are less than 0.05 , which have statistic significance

expression enhances metastasis of $\mathrm{BC}$ cells and results in poor survival of mice. Altogether, the above results demonstrate that miR-665 is required for $\mathrm{BC}$ cell migration and invasion in vitro, and metastasis in vivo, which is consistent with the clinical results. In general, epithelial-mesenchymal transition (EMT) is known as the first step of "invasion-metastasis cascade" ${ }^{25}$. Therefore, we asked if miR-665 could regulate EMT of BC cells. To this end, we first detected EMT protein markers by western blot analysis. The result showed that overexpressed miR665 indeed increased the expression of mesenchymal markers including $\mathrm{N}$-cadherin, $\beta$-catenin and Vimentin, and decreased epithelial marker E-cadherin in MCF-7 cells (Fig. 3g). To directly visualize the EMT, we performed immunofluorescence assay on EMT markers in BC cells. In MCF-7 cells with overexpressing miR-665, the levels of $\beta$-catenin and Vimentin were elevated and Ecadherin expression level was downregulated (Fig. 3f), suggesting that miR-665 may induce EMT before promoting the migration, invasion, and metastasis of $\mathrm{BC}$ cells. To further confirm that miR-665 expression induces EMT phenotype, we performed a dual-luciferase reporter assay on the promoter of $\beta$-catenin to verify miR-665 promoting $\beta$-catenin transcription. The result showed that the luciferase activity of the cells with miR665 overexpression was significantly higher than that in the control cells in both MCF-7 and MDA-MB-231 cells (Fig. 3h), suggesting that overexpressed miR-665 promotes transcriptional activity of $\beta$-catenin in $\mathrm{BC}$ cells. To theoretically verify that miR-665 can activate EMT pathway, we carried out gene set enrichment analysis (GSEA) based on ten pairs of BC samples with high and low miR-665 expressions (approximately eight-fold difference in average expression levels) obtained from TCGA database. Based on hallmark gene set, GSEA showed enrichment of genes involving in EMT in BC samples (Fig. 3i), which is consistent with the above experiment results. 
Table 4 Cox regression analysis of characteristics associated with survival of BC patients in FAHGMU cohort

\begin{tabular}{|c|c|c|c|c|}
\hline \multirow[t]{2}{*}{ Clinical characteristics } & \multicolumn{2}{|c|}{ Univariate Cox regression analysis } & \multicolumn{2}{|c|}{ Multivariate Cox regression analysis } \\
\hline & HR $(95 \% \mathrm{Cl})$ & $P$ Value & HR $(95 \% \mathrm{Cl})$ & $P$ Value \\
\hline \multicolumn{5}{|l|}{ Overall survival } \\
\hline miR-665 (high vs. low level) & $3.489(1.692-7.196)$ & 0.001 & $3.311(1.577-6.591)$ & 0.002 \\
\hline Age ( $\geq 50$ vs. $<50)$ & $1.433(0.747-2.746)$ & 0.279 & & \\
\hline Menopause (yes vs. no) & $1.413(0.746-2.676)$ & 0.289 & & \\
\hline Pathological Grade (III vs. I and II) & $1.314(0.549-3.145)$ & 0.539 & & \\
\hline Her-2 (positive vs. negative) & $0.774(0.404-1.484)$ & 0.440 & & \\
\hline PR (positive vs. negative) & $1.103(0.564-2.156)$ & 0.774 & & \\
\hline ER (positive vs. negative) & $0.771(0.405-1.470)$ & 0.430 & & \\
\hline TNBC (positive vs. negative) & $3.644(1.594-8.331)$ & 0.002 & $2.707(1.139-6.433)$ & 0.024 \\
\hline TNM stage (III vs. II vs. I) & $2.131(1.248-3.638)$ & 0.006 & $1.112(0.565-2.191)$ & 0.758 \\
\hline \multicolumn{5}{|l|}{ Disease-free survival } \\
\hline miR-665 (high vs. low level) & $3.546(1.779-7.068)$ & $<0.001$ & $3.278(1.619-6.636)$ & 0.001 \\
\hline Age ( $\geq 50$ vs. $<50)$ & $1.383(0.747-2.562)$ & 0.302 & & \\
\hline Menopause (yes vs. no) & $1.249(0.681-2.290)$ & 0.473 & & \\
\hline Pathological Grade (III vs. I \& II) & $1.685(0.780-3.640)$ & 0.184 & & \\
\hline Her-2 (positive vs. negative) & $0.764(0.413-1.415)$ & 0.392 & & \\
\hline PR (positive vs. negative) & $1.026(0.546-1.929)$ & 0.937 & & \\
\hline ER (positive vs. negative) & $0.814(0.439-1.508)$ & 0.513 & & \\
\hline TNBC (positive vs. negative) & $3.568(1.650-7.715)$ & 0.001 & $2.739(1.224-6.131)$ & 0.014 \\
\hline T stage (3-4 vs. 1-2) & $3.256(1.690-6.276)$ & $<0.001$ & 3.564 (1.590-7.988) & 0.002 \\
\hline N stage (2-3 vs. $0-1)$ & $2.332(1.212-4.488)$ & 0.011 & $2.430(1.087-5.435)$ & 0.031 \\
\hline TNM stage (III vs. II vs. I) & $2.007(1.211-3.326)$ & 0.007 & 1.107 (0.553-1.870) & 0.957 \\
\hline
\end{tabular}

The bold values are less than 0.05 , which have statistic significance

\section{NR4A3 is the target of miR-665 in breast cancer}

Given the above significant effects induced by miR-665 in $\mathrm{BC}$, we first need to decipher the target of miR-665 in BC. Thus, we set out to search the possible targets of miR665 on the web-based resources (https://mirmap.ezlab. org/app/ and http://www.mirdb.org). In both of databases, there are 459 shared predicted target genes of miR-665. According to miR-665 promotion of $\mathrm{BC}$ growth and metastasis, we selected 17 possible target genes to test which will be regulated by miR-665. In the BC cells overexpressing or underexpressing miR-665, we examined the mRNA expression of the 17 genes with qRTPCR. The result shows that of the 17 genes, only mRNA levels of NR4A3 and transforming growth factor beta receptor 2 (TGFBR2) were both decreased in cells overexpressing miR-665 and both increased in cells underexpressing miR-665 when compared with their corresponding control cells (Fig. S3B, C), which suggests that NR4A3 and TGFBR2 are the target of miR-665. Since TGFBR2 has been proved to be a target of miR-665 in pancreatic cancer and other cancer cells ${ }^{18,26}$, we only focued on the novel target, NR4A3, of miR-665 in BC.

Bioinformatics analysis showed that there is one seed sequence between 3' UTR of NR4A3 and miR-665 (Fig. 4a). To test if miR-665 can bind to $3^{\prime}$ UTR of NR4A3 via the seed sequences, we constructed wild type and mutated seed sequences in the $3^{\prime}$ UTR of NR4A3 mRNA (Fig. 4a). The $3^{\prime}$ UTR sequences was cloned into the Firefly luciferase reporter plasmid. Luciferase reporter experiment displayed that when MCF-7 or MDA-MB-231 cells were co-transfected with miR-665 mimics and the wild-type 3' UTR, the cells had significantly lower relative luciferase activity than the cells with miRNA scramble controls and the wild-type 3' UTR; when BC cells were co-transfected 
A

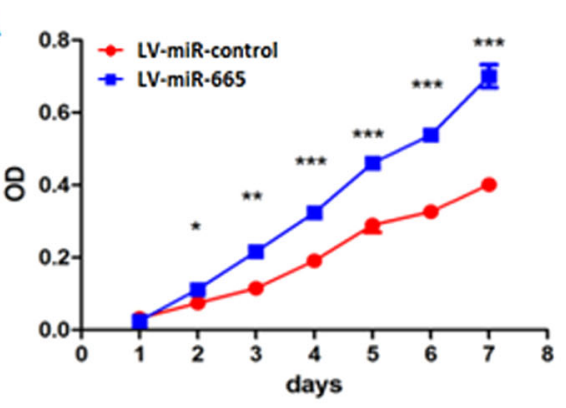

C

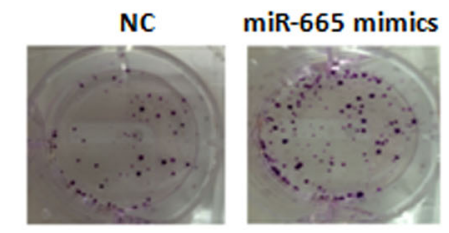

Inhibitor-control miR-665-inhibitor
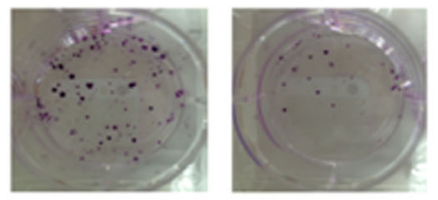

E

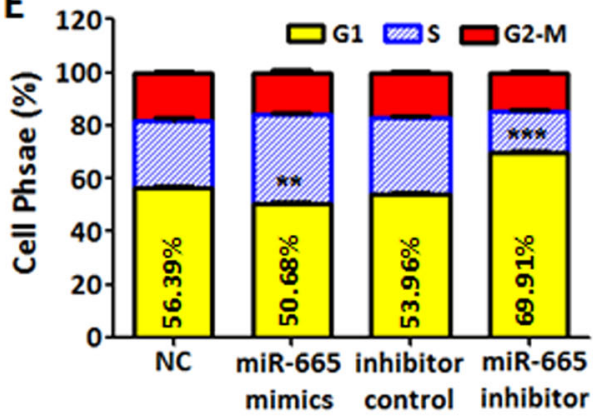

G

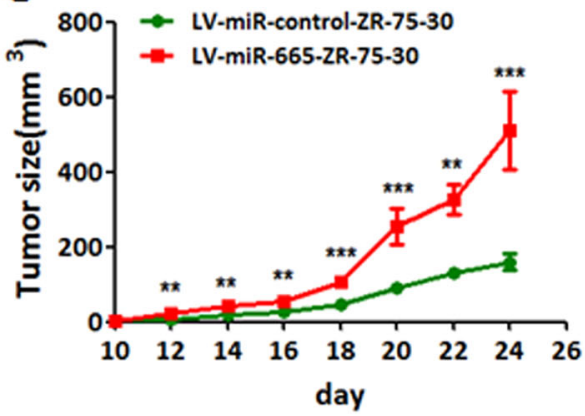

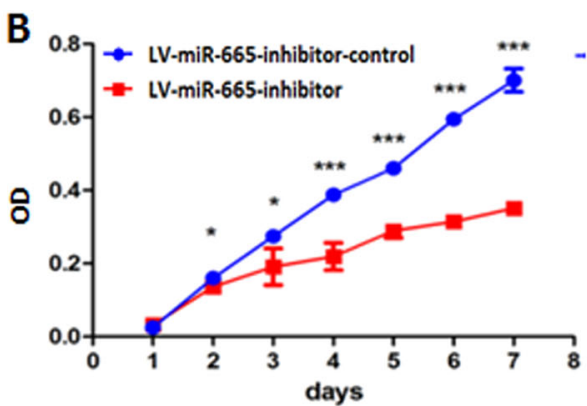

D

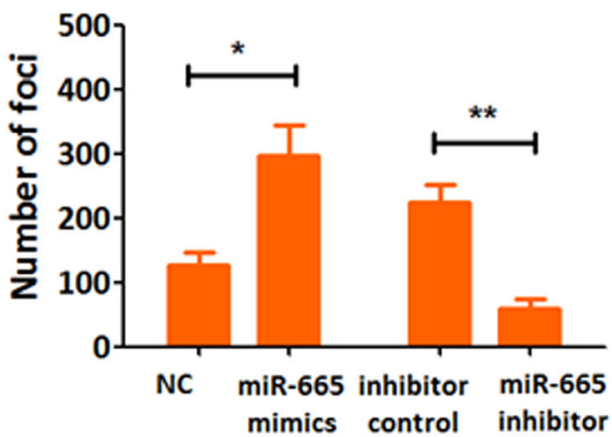

$\mathbf{F}$

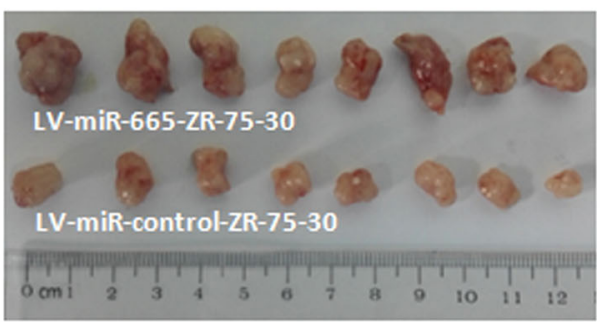

$\mathrm{H}$

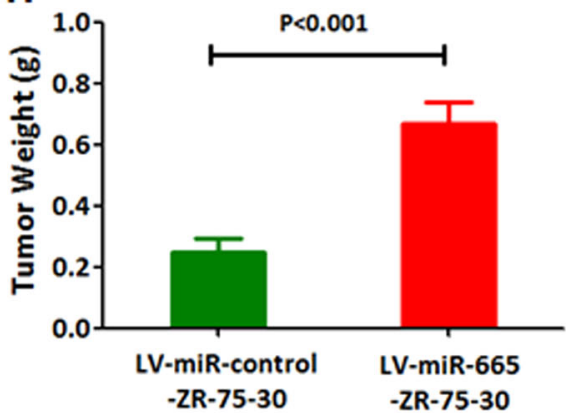

Fig. 2 miR-665 promotes BC cells proliferation and tumor growth. a Compared with the control MCF-7 cells stably expressing a scramble oligonucleotide, the MCF-7 cells stably expressing miR-665 have a much higher proliferation rate in cell counting kit-8 (CCK-8) assay. $\mathbf{b}$ In CCK-8 assay, MCF-7 cells stably expressing miR-665 inhibitor showed a significantly lower cell proliferation rate compared with the control MCF-7 cells. $\mathbf{c}$ In plate colony formation assay, the MCF-7 cells transfected with miR-665 mimics exhibited much more colonies while the MCF-7 cells transfected with miR665 inhibitor displayed much less colonies compared with their control cells, respectively. $\mathbf{d}$ The histogram is used to compare the colony numbers (average + standard deviation (SD)) from MCF-7 cells with different treatments in (c). e In the flow cytometry assay, the MCF-7 cells transfected with miR-665 mimics had the decreased G1 phase cells and the increased S phase cells while the MCF-7 cells transfected with miR-665 inhibitors were arrested in G1 phase (G1 phase cells were increased and S phase cells were decreased) compared with their control cells, respectively. $\mathbf{f}$ The subcutaneous xenograft tumors generated from LV-miR-665-ZR-75-30 cells (highly expressing miR-665) were significantly larger than those from control BC cells (LV-miR-control-ZR-75-30) in nude mice. $\mathbf{g}$ The tumor growth curves and $\mathbf{h}$ the average tumor weights and SDs in the two nude mice groups injected with ZR-75-30 cells stably expressing miR-665 or control oligonucleotide. $\left.{ }^{*} P<0.05,{ }^{* *} P<0.01,{ }^{* *} P<0.001\right)$ 

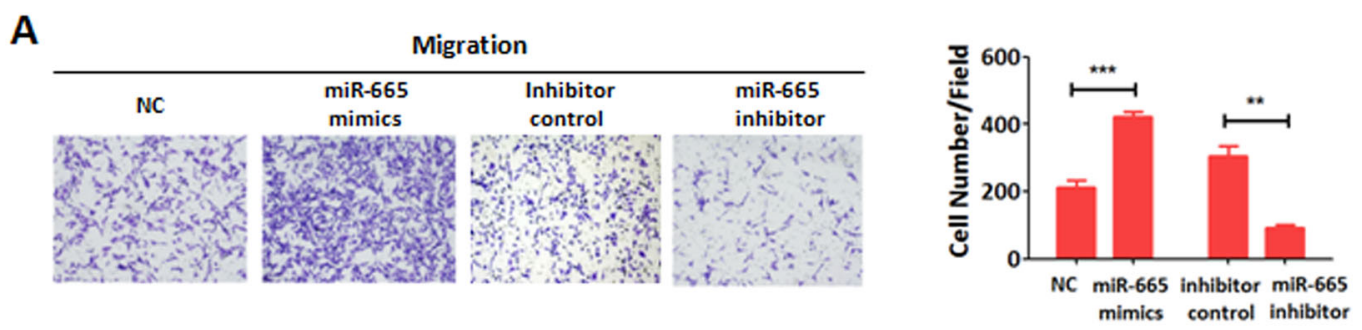

B
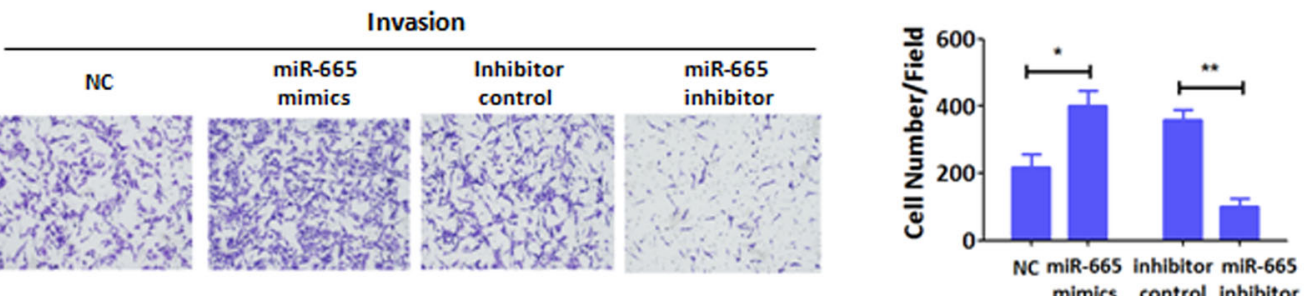

C

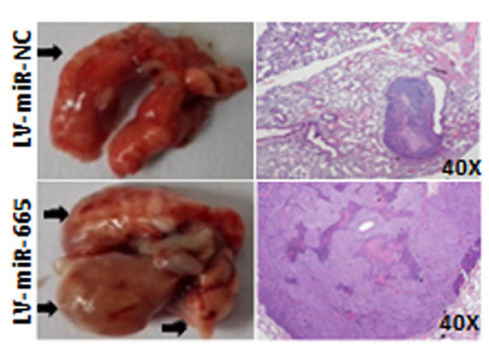

D

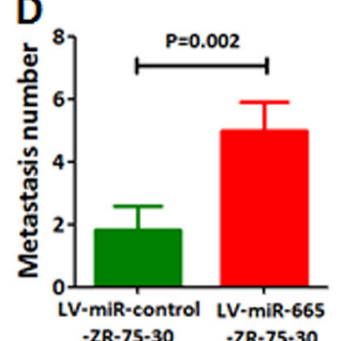

F

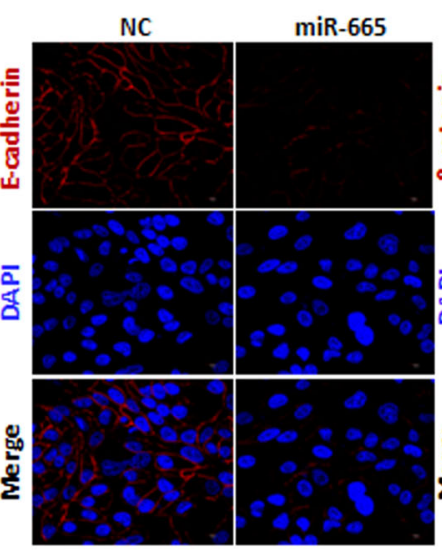

G

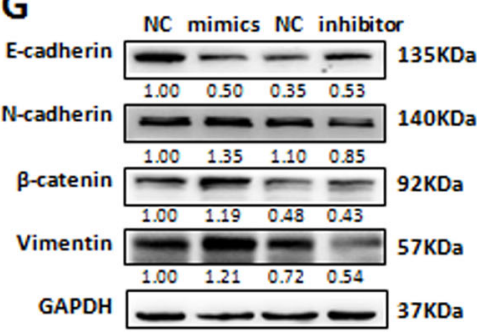

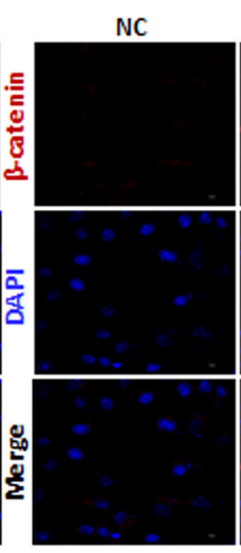

H

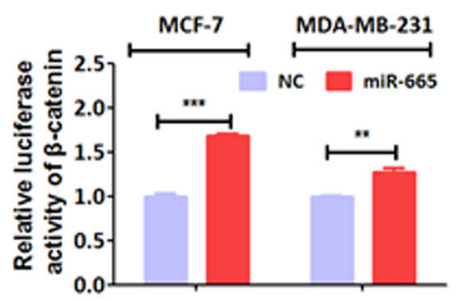

$\operatorname{miR}-665$
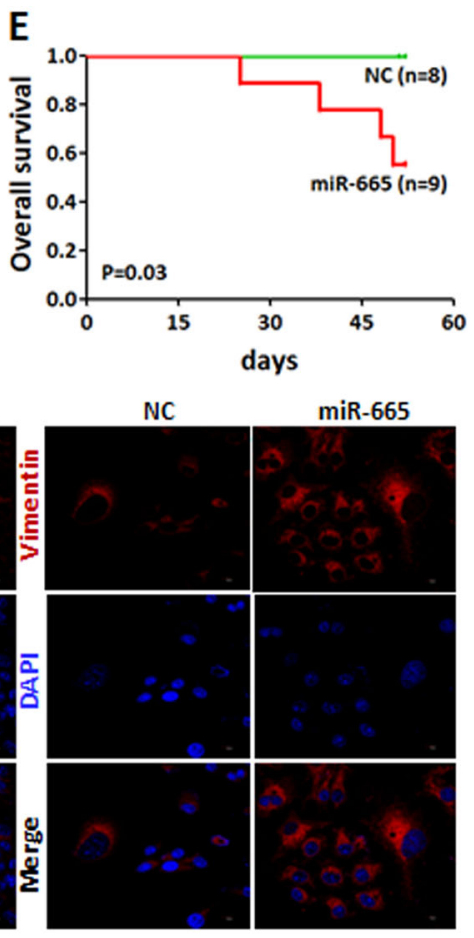

I

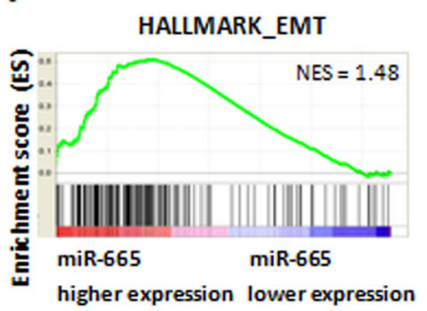

Fig. 3 (See legend on next page.) 


\begin{abstract}
(see figure on previous page)
Fig. 3 miR-665 promotes EMT, migration, invasion, and metastasis of BC cells in vitro and in vivo. a In transwell migration assay, MDA-MB-231 cells with transient transfection of miR-665 mimics showed more migrated cells and the cells with transient transfection of miR-665 inhibitors displayed much less migrated cells compared with their control cells, respectively (left panel); in the histogram, the numbers of migrated cells with different treatments in the left panel were compared (right panel). $\mathbf{b}$ In transwell invasion assay, MDA-MB-231 cells transfected with miR-665 mimics had more invaded cells and the cells transfected with miR-665 inhibitor had fewer invaded cells compared with their control cells, respectively (left panel); in the histogram, the numbers of invaded cells with different treatments in the left panel are compared (right panel). c Left column, the ZR-7530 cells expressing miR-665 or control oligonucleotide were injected into the tail veins of nude mice, and the lungs were resected at 50 day, in which the lung sample from mice with cells expression miR-665 had more metastatic nodules than those with control cells; right column, lung sections stained with haematoxylin and eosin exhibited a small metastatic focus from LV-miR-control-ZR-75-30 cells and a large tumor from LV-miR-665-ZR75-30 cells magnification $(\times 40)$. $\mathbf{d}$ The histogram was used to compare the average numbers of metastatic nodules in the lungs of the two mouse groups. (e) Survival curves of the two nude mouse groups, the mice injected with LV-miR-665-ZR-75-30 cells had significantly worse survival than the mice injected with LV-miR-control-ZR-75-30 cells. $\mathbf{f}$ In Immunofluorescence assay, MCF-7 cells treated with miR-665 NC or miR-665 mimics for 48 h, and the EMT protein markers were detected with the corresponding antibodies which is shown in red and DAPI is shown in blue. $\mathbf{g}$ In western blotting analysis, overexpression of miR-665 (mimics) decreased the level of epithelial protein maker (E-cadherin) and increased the levels of mesenchymal protein markers (N-cadherin, Vimentin, and $\beta$-catenin) in MCF-7 cells and the reverse results were observed in the cells with miR-665 downregulation (miR-665 inhibitor). h A dual-reporter (Gaussia Luciferase (GLuc) and Secreted Alkaline Phosphatase) system containing $\beta$-catenin promoter was co-transfected with miR-665 NC or miR-665 mimics into MCF-7 or MDA-MB-231 cells. Luciferase activity was detected after incubation for $48 \mathrm{~h}$ and data represent mean \pm SD compared to control cells $(n=3)$, which indicated that when miR-665 was ectopically expressed in BC cells, $\beta$-catenin transcriptional activity was increased. i Gene set enrichment analysis (GSEA) based on ten patients with low-miR-665 expression (blue) and ten those with high-miR-665 expression (red) showed that miR-665 high-expression was involved in the in EMT pathway activation. NES means normalized enrichment score. ${ }^{*} P<0.05,{ }^{* *} P<0.01,{ }^{* * *} P<0.001$, independent Student's $t$ test
\end{abstract}

with miR-665 mimics and the mutated 3' UTR, the cells had nearly the same relative luciferase activity as the control cells (Fig. 4b), which demonstrate that wild type miR-665 can bind to the seed sequences of the 3' UTR of NR4A3 mRNA to inhibit translation and activity of luciferase whereas it can not bind to the mutated seed sequences so that luciferase activity is not affected. Furthermore, we employed qRT-PCR and western blot to examine whether the mRNA and protein of NR4A3 were inhibited by miR-665. The result reveals that the cells overexpressing miR-665 show an obvious downregulation of NR4A3 mRNA and protein whereas the cells underexpressing miR-665 exhibit upregulated levels of NR4A3 mRNA and protein when compared with the corresponding control cells in MCF-7 and MDA-MB-231 cells (Fig. 4c, d). Altogether, these results demonstrate that NR4A3 is a direct target of miR-665.

To further confirm that NR4A3 is a target of miR-665 in $\mathrm{BC}$ tissues, we explored the relationship between the expression levels of miR-665 and its target gene in $\mathrm{BC}$ samples. First, we used qRT-PCR to examine the RNA expression levels of miR-665 and NR4A3 in tumor tissues of $\mathrm{BC}$ patients. The result showed that NR4A3 mRNA levels in clinical $\mathrm{BC}$ tissues $(N=15)$ is significantly lower than the normal breast tissues $(N=15)$ (Fig. 4e) and that the mRNA level of NR4A3 was reversely correlated with the expression levels of miR-665 in these samples $(R=$ $-0.4147, P=0.001$ ) (Fig. 4f). Second, we investigated NR4A3 expression with immunohistochemical staining in xenograft tumors generated from BC cells overexpressing miR-665 (LV-miR-665-ZR-70-30 cells), and found that NR4A3 was downregulated in these tumors compared with that in the control tumors generated from LV-miRcontrol-ZR-70-30 cells (Fig S4A). These results clearly suggest that NR4A3 is the target of miR-665 in BC.

\section{miR-665 promotes $B C$ invasion and metastasis by inhibiting NR4A3}

It is known that NR4A3 is a transcriptional activator and plays important roles in many physiological and pathological processes ${ }^{27}$. More important, NR4A3 is involved in the development and progression of various tumors including the primary extraskeletal myxoid chondrosarcoma, lymphoma, acute myeloid leukemia and gastric cancer, and functions as a tumor suppressor ${ }^{27-31}$. Based on our above results that miR-665 targets NR4A3 and can promote EMT, migration, invasion, and metastasis of BC cells in vitro and in vivo, we argued that miR665 advances the metastasis of BC by targeting NR4A3. To this end, using wound healing assay in MDA-MB-231, we first examined if silenced NR4A3 would promote BC migration as overexpressed miR-665 did. The result proved that the wound healing was significantly accelerated in NR4A3-silenced MDA-MB-231 cells when compared with the control cells (Fig. 5a, b). Secondly, matrigel invasion assay also showed that the number of invaded MDA-MB-231 cells with silenced NR4A3 was significantly more than that of invaded control cells (Fig. $5 \mathrm{c}$, d). These results indicate that silenced NR4A3 induces the same phenotypes of BC cells as miR-665 expression does, suggesting that miR-665 promotes migration and invasion of $\mathrm{BC}$ cells by targeting NR4A3. To substantiate our hypothesis, we conducted a rescue experiment in MDAMB-231 cells. The BC cells depleted of endogenous miR- 

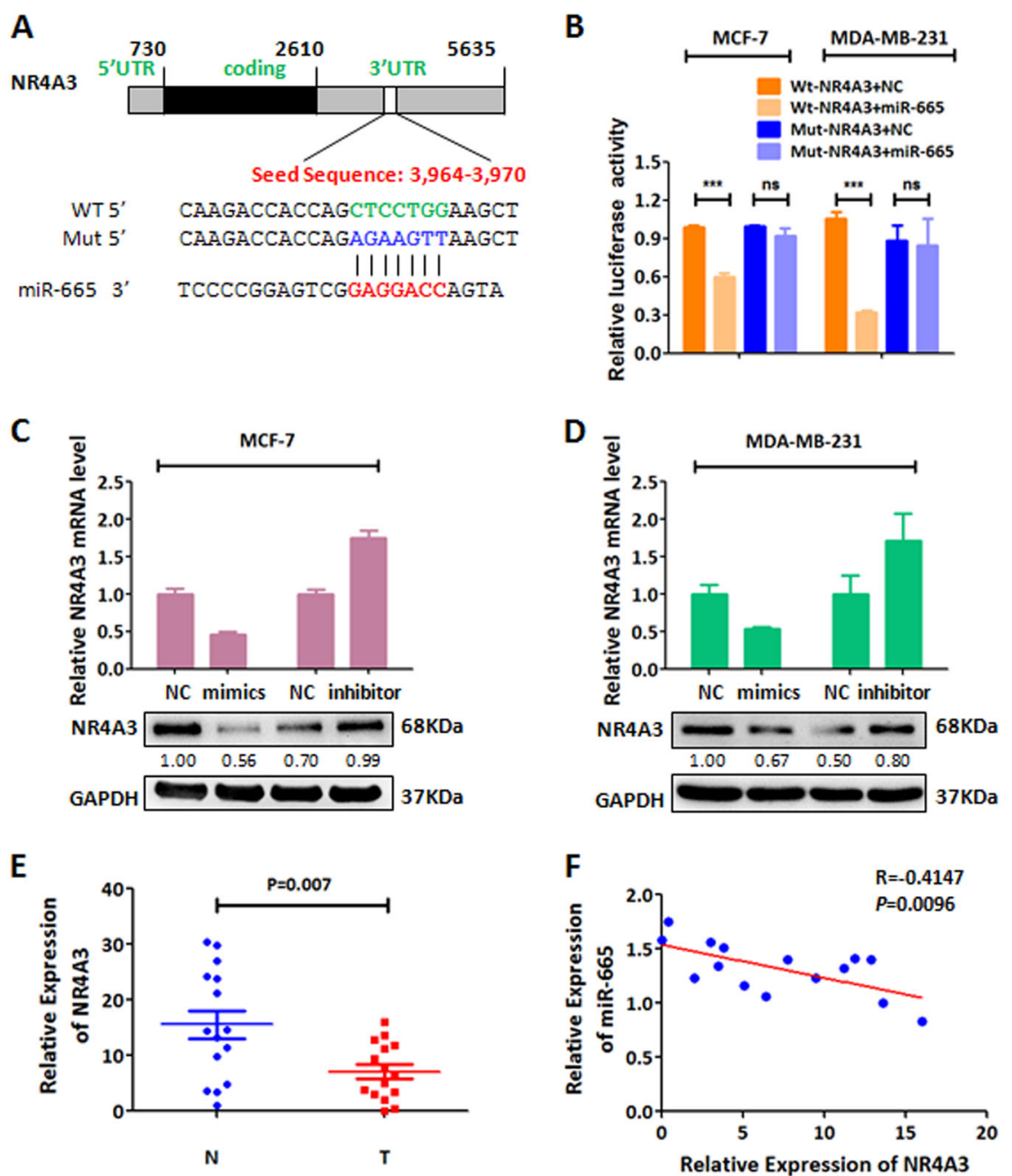

Fig. 4 miR-665 targets NR4A3 in BC cells. a The binding seed sequence (in green) of miR-665 in 3'UTR of NR4A3 mRNA as predicted by the TargetScan algorithm, and the artificially mutated seed sequence (in blue) in the same $3^{\prime} U T R$, and the seed sequence in mir-665 (in red). $\mathbf{b}$ In dualluciferase assay, MCF-7 or MDA-MB-231 cells co-transfected with reporter vector containing wild type 3'UTR of NR4A3 and miR-665 mimics showed a significant reduction in luciferase activities compared with the control cells co-transfected with the wild-type $3^{\prime} U T R$ and control oligonucleotide (NC), while in these cells co-transfected with the mutated $3^{\prime} U T R$ of NR4A3 and miR-665 mimics, there was no reduction in luciferase activities compared with the control cells. c-d In qRT-PCR and western blotting analysis, MCF-7 (c) and MDA-MB-231 (d) cells transfected with miR-665 mimics exhibited Iow mRNA (upper panel) and protein (lower panel) levels of NR4A3; when these cells were transfected with miR-665 inhibitor, the mRNA (upper panel) and protein (lower panel) levels of NR4A3 were increased compared with the control cells. e The relative mRNA expression of NR4A3 detected by qRT-PCR was significantly lower in BC tissues (15 samples) in comparison with normal breast tissues (15 samples, $P<0.01$, independent Student's $t$ test). $\mathbf{f}$ An inverse correlation was found between miR-665 expression and NR4A3 mRNA expression in 15 BC tissues, as examined by qRT-PCR ( $R=$ $-0.4147, P=0.001)$

665 by miR-665 inhibitor were incubated for $36 \mathrm{~h}$ followed by depletion of NR4A3 using a siRNA aginst NR4A3 for another $24 \mathrm{~h}$. As shown in the above experiments, we demonstrated that depletion of endogenous miR-665 with miR-665 inhibitor significantly inhibited the migration and invasion of MDA-MB-231 cells compared with the control cells in wound healing assay and matrigel invasion assay (Fig. $5 \mathrm{e}-\mathrm{g}$ ), respectively. In this rescue experiment, when MDA-MB-231 cells were transfected with siRNA against NR4A3, the cell migration and invasion were increased compared with the control cells; when the cells were sequentially depleted of miR-665 and NR4A3, the inhibited cells again restored their motility and invasiveness by exhibiting accelerated wound healing and increased invasion compared with the corresponding control cells (miR-inhibitor + siRNA-NC treatment) (Fig. $5 f$, h). Furthermore, we conducted the same rescue experiment in MCF-7 and MDA-MB-231 cells and 


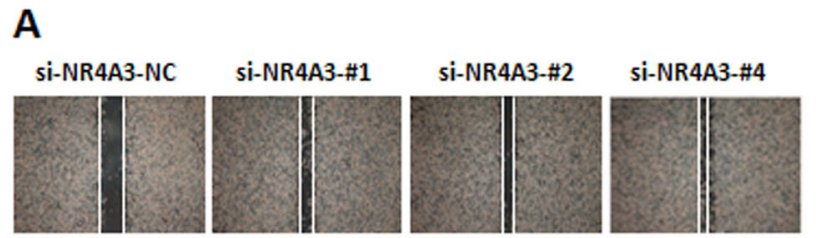

C

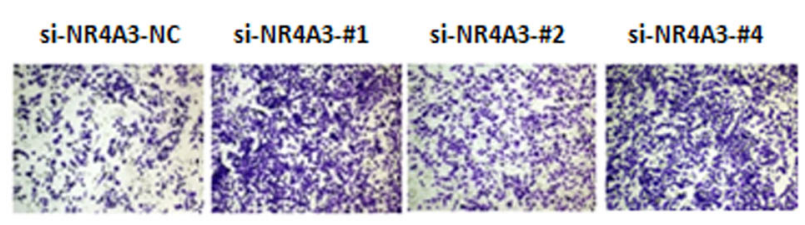

B

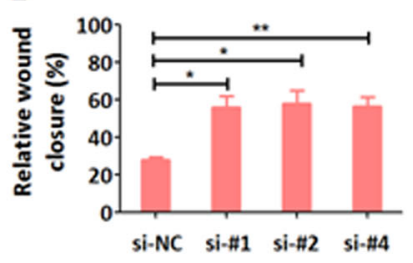

D

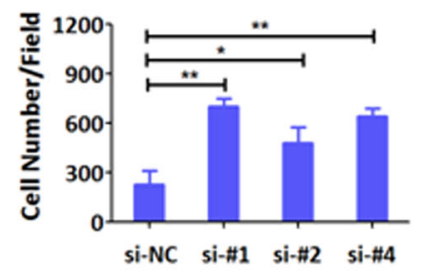

E

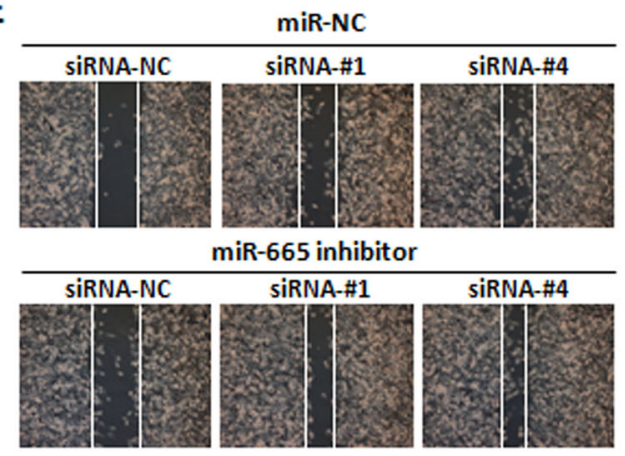

G

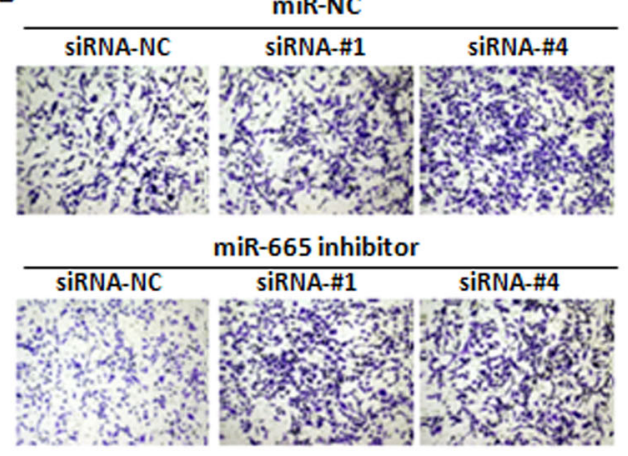

$\mathbf{F}$

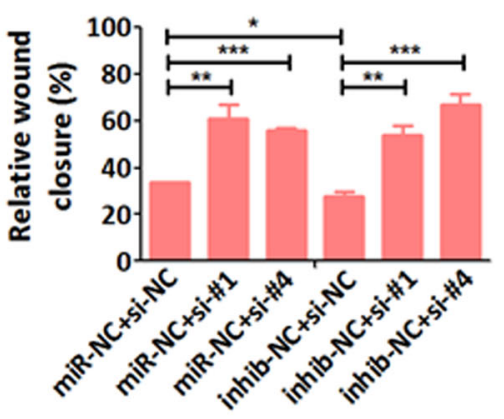

H

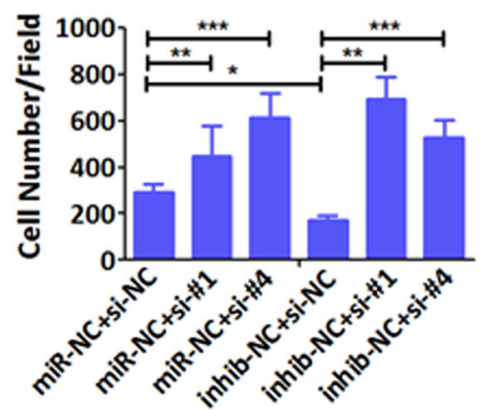

Fig. 5 miR-665 enhances migration and invasion of BC cell by targeting NR4A3. a In scratch wound healing assay, MDA-MB-231 cells with transient transfection of NR4A3 siRNA \#1, \#2, \#4 showed a significantly accelerated wound healing compared with the control cells treated by NR4A3 siRNA-NC. $\mathbf{b}$ In the histogram, the percentages of wound healing of the cells with different treatments in (a) were compared. $\mathbf{c}$ In transwell invasion assay, MDA-MB-231 cells with siRNA \#1, \#2, \#4 against NR4A3 had more invaded cells. $\mathbf{d}$ In the histogram, the numbers of invaded cells with different treatments in (c) were compared. e In scratch wound healing assay, after MDA-MB-231 cells were treated with miR-665 inhibitor or miR-NC for $36 \mathrm{~h}$ and then with NR4A3 siRNA-NC, \#1 or \#4 for another $24 \mathrm{~h}$, respectively, the cells with miR-665 inhibitor and siRNA \#1 or \#4 had an accelerated (rescued) wound healing compared with those with miR-665 inhibitor and siRNA-NC. $\mathbf{f}$ In the histogram, the percentages of the wound healing in the cells with different treatments in (e) were compared. $\mathbf{g}$ In transwell invasion assay, after MDA-MB-231 cells were transfected with miR-665 inhibitor or miR-NC for $36 \mathrm{~h}$ and then with NR4A3 siRNA-NC, \#1 or \#4 for another $24 \mathrm{~h}$, respectively, the cells with miR-665 inhibitor and siRNA \#1 or \#4 had a greater number of invaded cells (rescued) compared with those with miR-665 inhibitor and siRNA-NC. $\mathbf{h}$ In the histogram, the numbers of invaded cells with different treatments in $(\mathbf{g})$ were compared. In this figure, results were expressed as the mean \pm SD of three independent experiments. ${ }^{*} P<0.05,{ }^{* *} P<0.01,{ }^{* * *} P<0.001$ by Student's $t$ test 


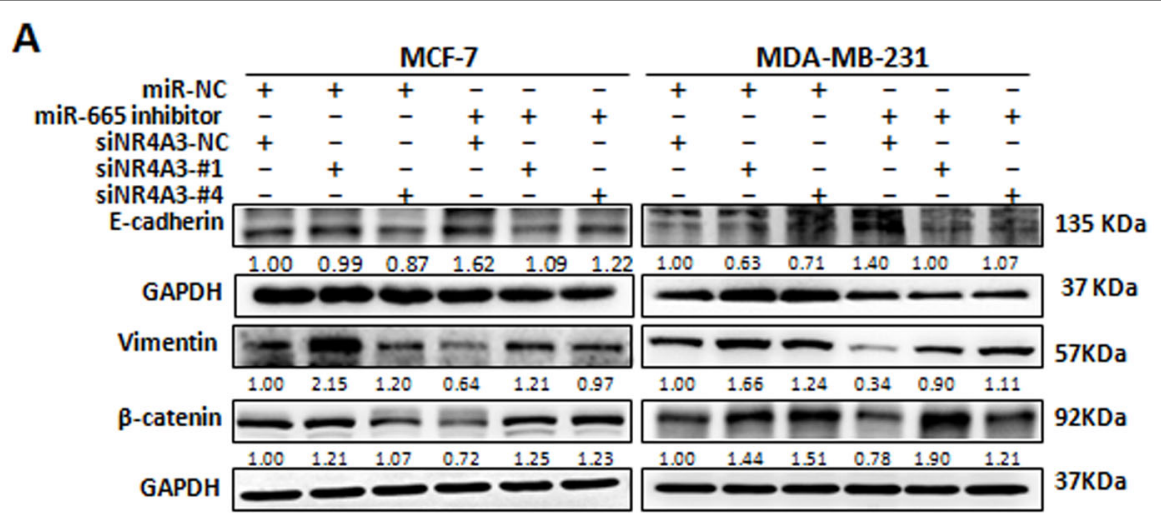

B

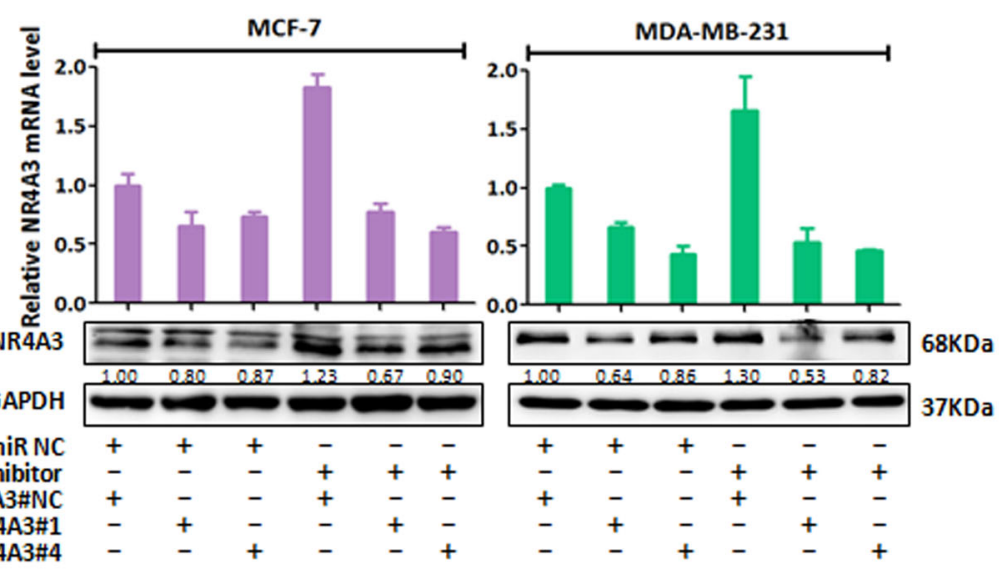

C

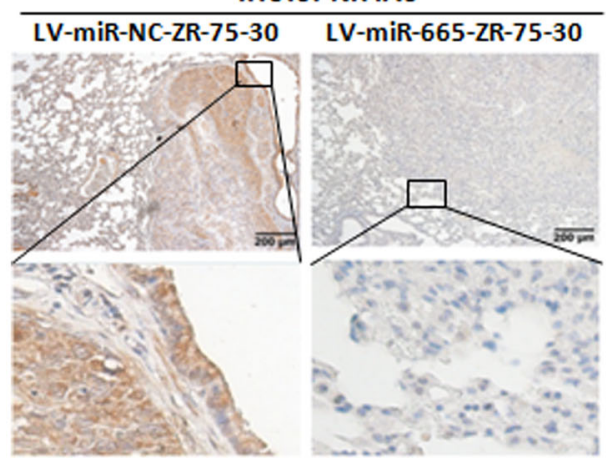

E

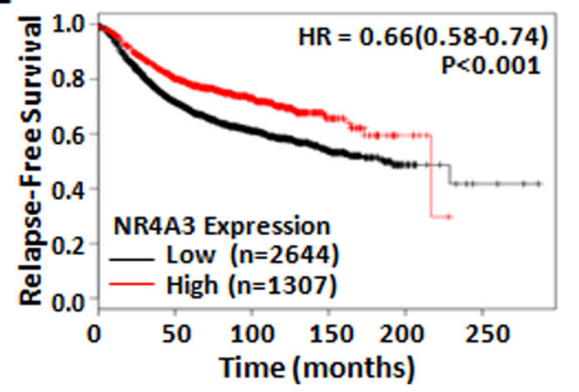

D
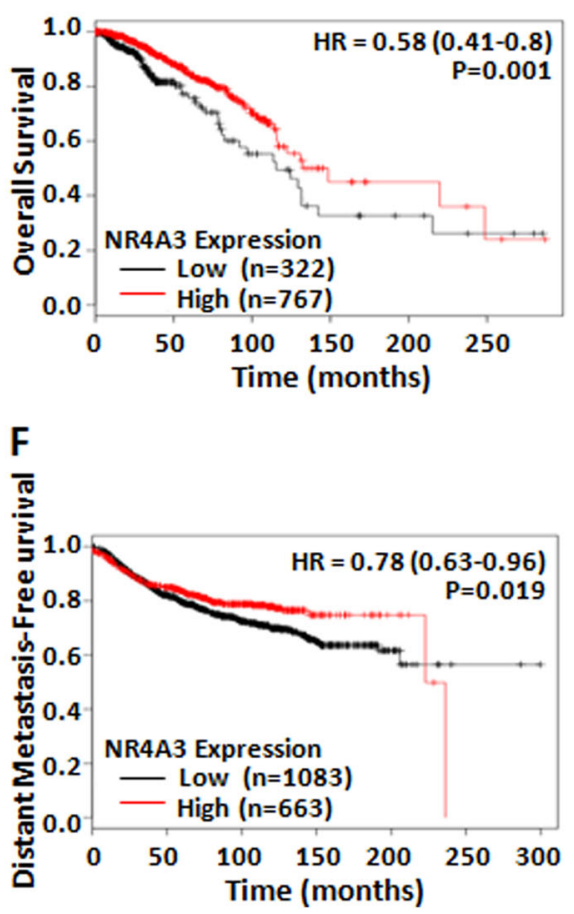

Fig. 6 (See legend on next page.) 


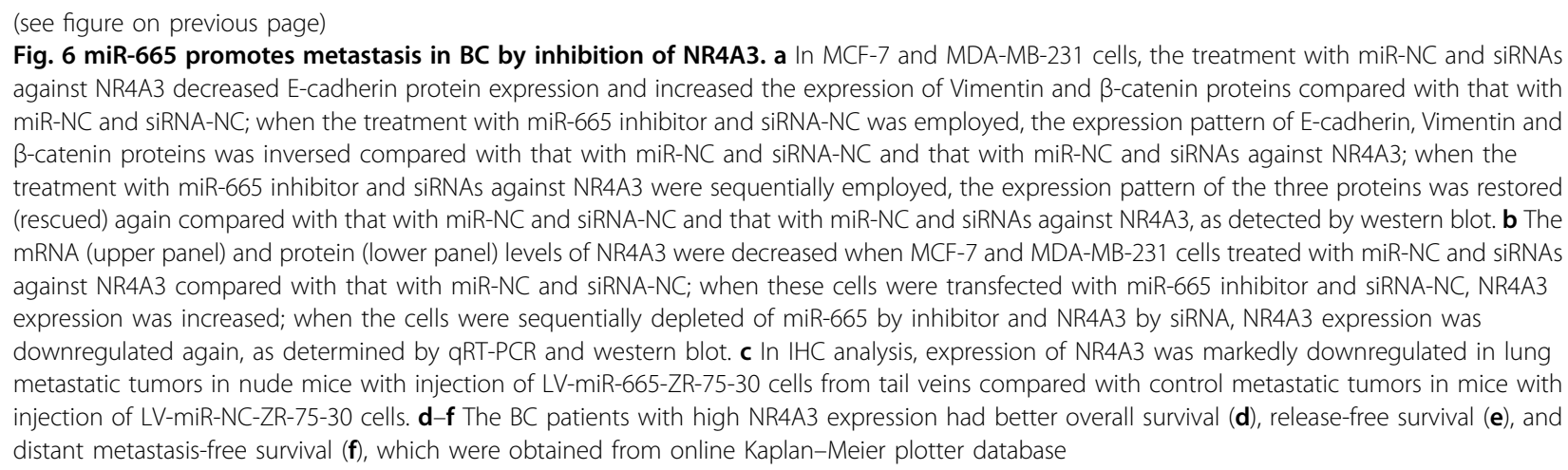

observed EMT changes with western blot. As showed in Fig. 6a, when miR-665 expression was inhibited with miR665 inhibitor, the expression of mesenchymal markers (Vimentin and $\beta$-catenin) was decreased and epithelial marker (E-cadherin) was increased compared with the control treatment in MCF-7 and MDA-MB-231 cells; when NR4A3 was depleted with siRNAs, the expression pattern of the three EMT markers was inversed in these cells; when the expressions of miR-665 and NR4A3 were inhibited sequentially with miR-665 inhibitor and siRNA, the expression pattern of three EMT markers was rescued again, suggesting that miR-665 promotes EMT by targeting NR4A3. At the same time, we examined mRNA and protein expression of NR4A3 by qRT-PCR and western blot when the $\mathrm{BC}$ cells were treated with miR-665 inhibitor and siRNAs against NR4A3. The result shows that the mRNA and protein expression of NR4A3 were increased when $\mathrm{BC}$ cells were treated with miR-665 inhibitor, whereas they were decreased when these $\mathrm{BC}$ cells were transfected again with siRNAs against NR4A3 (Fig. 6b).

As mentioned above, overexpressed miR-665 promoted lung metastasis of $\mathrm{BC}$ cells in nude mice. We wondered if NR4A3 was involved in the metastasis of BC. Thus, we investigated NR4A3 protein level in the metastatic nodules with immunohistochemistry (IHC) and found that NR4A3 was significantly reduced in the lung metastatic BC nodules from BC cells with upregulated miR665 (Fig. 6c). In the TCGA database, NR4A3 expression in breast cancers is remarkably reduced compared with that in normal breast tissues (Fig. S4B). Furthermore, we explored the KM Plotter Database and found that patients with high-expression of NR4A3 had significantly better OS, RFS, and DMFS than those with low expression of NR4A3 (Fig. 6d-f), suggesting that NR4A3 expression inhibits metastasis and progression of $\mathrm{BC}$ in these patients. Altogether, these results undoubtedly demonstrate that miR-665 promotes invasion and metastasis by inhibiting NR4A3.

\section{miR-665 enhances metastatic phenotype in BC via NR4A3/ MEK pathway}

To understand the mechanism underlying miR-665 promotion of BC metastasis, we carried out GSEA as described above. Based on KEGG pathway and oncogenic signature/hallmark gene set, GSEA showed enrichment of genes involving in cell cycle, TGFB, MEK, and other pathways in high vs. low level of miR-665 in BC samples (Fig. 7a), the EMT and cell cycle of which have been verified in our above experiments. Since TGFB signaling and its upstream gene TGFBR2, which has been reported to be a target gene of miR-665 in pancreatic cancer and other cancer cells ${ }^{18,26}$, have been reported in $\mathrm{BC}^{32,33}$, we interested in whether miR-665 promotes $\mathrm{BC}$ metastasis by activating MEK signaling, which is reported to advance $\mathrm{BC}$ metastasis ${ }^{34,35}$. Thus, we investigated the downstream target ERK and Slug of MEK pathway in BC cells with miR-665 overexpression or underexpression. The result indicates that both Slug expression and ERK phosphorylation were increased in MCF-7 and MDA-MB-231 cells with miR-665 overexpression and decreased in $\mathrm{BC}$ cells with less expression of miR-665 when compared with the corresponding control cells (Fig. 7b), suggesting that miR-665 promotes migration, invasion and metastasis via activating MEK pathway. Then the key question is whether miR-665 activates MEK signaling by inhibiting NR4A3. To this purpose, we observed expression changes of NR4A3, Slug, and ERK under different doses of miR-665 mimics in BC cells. As expected, the expression of Slug, $\beta$-catenin, and Vimentin as well as ERK phosphorylation were gradually upregulated, and NR4A3 and E-cadherin were downregulated as the dose of miR-665 mimics was increased from 5, 50 to $100 \mathrm{nM}$ (Fig. 7c). To further corroborate that miR-665 activates EMT and MEK pathways via inhibiting NR4A3, we conducted another rescue experiment: when only miR-665 was downregulated by miR-665 inhibitor, Slug expression and ERK phosphorylation were decreased; when only NR4A3 was downregulated by siRNAs, Slug expression and ERK phosphorylation also were inversed; 

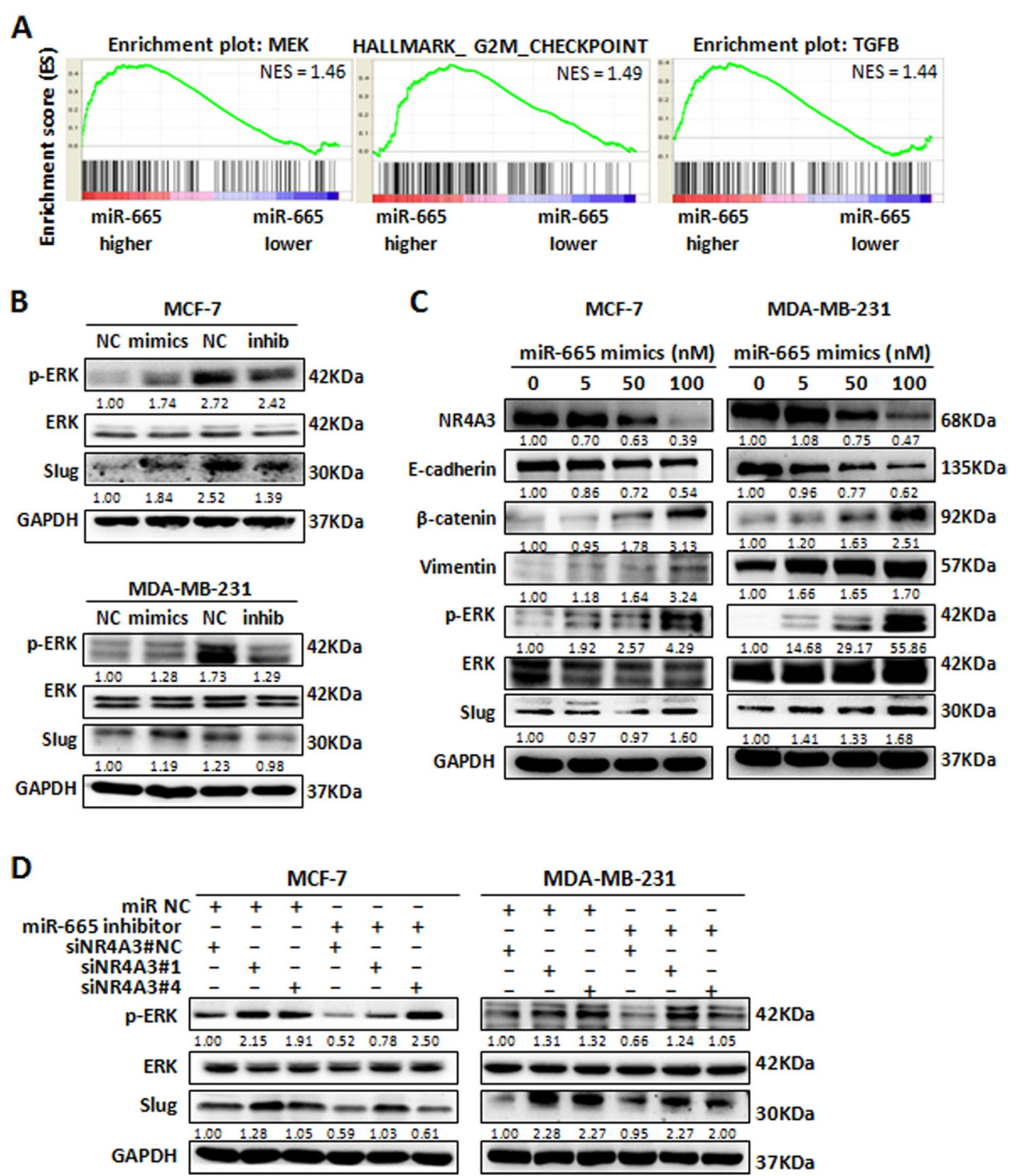

Fig. 7 miR-665 expression contributes a more aggressive metastatic phenotype in BC via activation of NR4A3/MEK pathway. a GSEA analysis was performed in 10 pairs of BC tissues with miR-665 high expression (red) and low expression (blue) obtained from TCGA database, and the result showed that miR-665 high-expression was involved in activation of multiple signaling pathways, such as MEK, EMT, G2M, and TGFB pathways. NES normalized enrichment score. $\mathbf{b}$ In MCF-7 and MDA-MB-231 cells, western blotting analysis indicated that transfection of miR-665 mimics increased the level of ERK phosphorylation and Slug expression, whereas transfection of miR-665 inhibitors showed the reverse results compared with the corresponding control treatments. c In MCF-7 and MDA-MB-231 cells, when the cells were transfected with different doses of miR-665 mimics, the expression levels of NR4A3 and E-cadherin were gradually decreased while the expression of $\beta$-catenin, Vimentin, phosphorylated ERK ( $p$ ERK), and Slug were increased following the increased doses of miR-665 mimics, as measured by western blot. $\mathbf{d}$ In MCF-7 and MDA-MB-231 cells, western blotting analysis showed that the protein levels of p-ERK and Slug were increased when the cells were depleted of NR4A3; when only miR665 was downregulated by miR-665 inhibitor, the protein levels were decreased; when the cells were treated by sequential depletion of miR-665 and NR4A3, the p-ERK and Slug were increased (rescued) again. The values above the blot bands are the relative digital intesnsity of the bands

when miR-665 and NR4A3 were downregulated sequentially, Slug expression and ERK phosphorylation were rescued again (Fig. 7d). The results indicate that NR4A3 represses MEK pathway including ERK phosphorylation and Slug expression, consistent with the report by Rodríguez group that NR4A3 inhibits ERK phosphorylation ${ }^{36}$. Altogether, our results demonstrate that upregulated miR-
665 activates MEK pathway by inhibiting NR4A3 to promote EMT, migration, invasion, and metastasis of $\mathrm{BC}$ cells.

\section{Discussion}

In this study, we profiled miRNA expression in $422 \mathrm{BC}$ patients from Southern China using a custom microarray, and found that 399 miRNAs were differentially expressed 
between $\mathrm{BC}$ and noncancerous tissues. Among the upregulated miRNAs, miR-665 was highly expressed in $\mathrm{BC}$ tissues and associated with poor OS, DFS, and DMFS of $\mathrm{BC}$ patients. High-expression level of miR-665 also was found to be positively related to $\mathrm{T}$ stage, $\mathrm{N}$ stage, distant metastasis as well as TNM stage. The correlation between overexpressed miR-665 measured by qRT-PCR and poor survival was validated in $161 \mathrm{BC}$ samples obtained from another medical center in South China. Furthermore, this correlation was corroborated in total $2323 \mathrm{BC}$ patients from other parts of the world. The clinical data indicate that miR-665 is a pro-oncogenic regulator and promotes the development and progression of $\mathrm{BC}$, especially tumor metastasis, suggesting that miR-665 is a potential biomarker for predicting survival and metastasis of $\mathrm{BC}$.

Like many other miRNAs, miR-665 has been reported to show pro-oncogenic and tumor suppressive roles in different types of cancers because it suppress different target genes, oncogenes or tumor suppressors in different cancers $^{37}$. As a tumor suppressor, miR-665 is downregulated in osteosarcoma tissues and represses metastasis of osteosarcoma cells by inhibiting Rab23 and TGFBR2 ${ }^{17,26}$; miR-665 expression also is reduced in lung cancer and gastrointestinal stromal tumor cells and targets MAPK signaling pathways and $\mathrm{CD} 34^{38,39}$; in pancreatic cancer cells, miR-665 is downregulated and inhibits oncogenic phenotype by targeting TGFBR 1 and TGFBR $2^{18}$; in ovarian cancer cells, miR-665 is downregulated and can suppress tumor growth and migration $^{40}$; in mouse model, miR-665 can repress neuroblastoma cell growth by targeting c-myc and HDAC ${ }^{41}$. However, miR-665 is also reported to be upregulated in HCC tissues, HCC cell lines and serum exosome of HCC patients, and the overexpressed miR665 promotes $\mathrm{HCC}$ cell proliferation, migration, and invasion by inhibiting PTPRB ${ }^{14,42}$; a high level of miR-665 is associated with resistance to neoadjuvant radiochemotherapy in squamous cell carcinoma of the esophagus $^{43}$; in nonsmall cell lung cancer, miR-665 is overexpressed in plasma extracellular vesicles of patients and lung cancer cell lines ${ }^{15}$, suggesting that miR-665 acts as an oncogene. In a word, miR-665 can act as either a tumor suppressor or an oncogene depending on the cancer type or the cellular content. Interestingly, one group reported that miR-665 was downregulated in $48 \mathrm{BC}$ tissue samples compared with the corresponding adjacent breast tissues ${ }^{19}$, which is inconsistent with our result. The major reason might be the different sample sizes (422 BCs vs. $48 \mathrm{BCs}$ ) in our study and the others'.

In the present study, we demonstrated the oncogenic role of miR-665 in breast tumorigenesis and tumor metastasis by gain- and loss-of function experiment in vitro and in vivo. Notably, overexpression of miR-665 promotes migration and invasion of MDA-MB-231 cells and advances lung metastasis of $\mathrm{BC}$ cells in nude mice. GSEA analysis shows that high-expression of miR-665 enriched genes involving in EMT, suggesting that miR665 can induce EMT. Therefore, we examined the transcriptional activity of $\beta$-catenin, a representational EMT marker, in BC cells expressing miR-665. The result shows that overexpressed miR-665 can upregulated the luciferase activity of $\beta$-catenin promoter in BC cells significantly, which suggests that by promoting $\beta$-catenin transcriptional activity, miR-665 may promote the EMT in BC. More important, we demonstrated that miR-665 promotes EMT, migration, invasion and metastasis of $\mathrm{BC}$ cells through targeting NR4A3. Firstly, ectopic expression of miR-665 decreases the mRNA and protein levels of NR4A3 in BC cells, and the downregulated miR-665 increases the expression of this gene. Secondly, miR-665 can bind to the 3' UTR of NR4A3 as evidenced by luciferase reporter assay and bioinformatic analysis.

NR4A3 is a member of the steroid-thyroid hormoneretinoid receptor superfamily and is demonstrated to be a transcriptional activator that can efficiently bind to the NGFI-B response element (NBRE) ${ }^{44,45}$. Previous studies have suggested that NR4A3 acts as a tumor suppressor in tumorigenesis such as hematologic neoplasms, acute myeloid leukemia, lymphoma, and gastric cancer by reducing cell viability and inducing cell apoptosis ${ }^{27,30,31}$. In breast cancer, NR4A3 is downregulated, and functions as a tumor suppressor $^{46}$, which is consistent with our result. In TCGA database, NR4A3 expression also dramatically reduced in $\mathrm{BC}$ tissues compared with normal breast tissues (Fig. S5B). In the present study, we found that NR4A3 was downregulated whereas miR-665 was upregulated in BC tissues, which were significantly inversely correlated with each other. In in vitro experiment, downregulation of NR4A3 markedly restored the EMT, migration, and invasion of $\mathrm{BC}$ cells inhibited by miR-665 inhibitor; in BC metastasis model in nude mice, NR4A3 is significantly reduced in the metastatic nodules formed from BC cells with miR-665 overexpression (Fig. 6c), indicating that miR-665 promotes EMT, migration, invasion, and metastasis of $\mathrm{BC}$ cells by targeting NR4A3. To explore the underlying the mechanism, we conducted a GSEA in TCGA database, which showed that high expression of miR-665 enriched genes involving in MEK, and other pathways, of which MEK is reported to advance $\mathrm{BC}$ metastasis ${ }^{34,35}$. Therefore, we mainly investigated whether miR-665 could activate MEK signaling in BC cells. The result indicates that miR-665 can regulated both Slug expression and ERK phosphorylation of MEK signaling cascade in MCF-7 and MDA-MB-231 cells (Fig. 7b). Next, we demonstrated that NR4A3 expression inhibited MEK pathway including ERK phosphorylation and Slug expression in BC cells. Finally, we showed that miR-665 activates MEK pathway by inhibiting NR4A3. These results indicate that miR-665 activated MEK 
signaling pathway via inhibiting NR4A3, by which miR-665 promotes metastasis phenotype of $\mathrm{BC}$ cells.

In this study, we demonstrate for the first time that miR-665 expression is associated with metastasis and poor survival in $\mathrm{BC}$ patients and plays an oncogenic role in $\mathrm{BC}$ progression with clinical analysis and both in vivo and in vitro experiments. Furthermore, we elucidate that miR-665 promotes $\mathrm{BC}$ invasion and metastasis via targeting NR4A3 to activate MEK signaling pathway. Altogether, our study provides the evidence that miR-665 is a potential new biomarker for recurrence, metastasis, and survival, and therapeutic target in patients with $\mathrm{BC}$.

\section{Acknowledgements}

We are grateful to Dr. Yibin Kang, a professor of Princeton University, for his critically reviewing and revising the manuscript. And we also thank Dr. Qi Wang, Pharm. D., a Medical student of 2015 class in Rutgers University, New Jersey, USA, for her proof-reading! This research was supported by grants from National Natural Science Foundation of China/Joint Research Fund for Overseas Chinese Scholars and Scholars in Hong Kong and Macao (Grant nos. 81228104 and 81629004 ) and partially supported by grants from National Natural Science Foundation of China (Grant no. 81372564, 81572466, and 81772991). The funding bodies played no role in the design, collection, analysis, or interpretation of the data; in the writing of the paper; or in the decision to submit the paper for publication.

\section{Author contributions}

H.Y.W., J.Y.H. and X.G.Z. designed the study. J.T., W.Y., R.D., H.Z.Z. and J.H.H. collected the tissue samples and interpreted the clinical data. H.Z.Z. and J.H.H. performed the pathological examination of breast cancer tissue sections. J.Y.H., X.G.Z., M.Y.Z., N.Q.W., R.Q.W. and J.L. performed the experiments and statistical analysis. H.Y.W., J.Y.H. and X.G.Z. wrote the draft of the paper. H.Y.W. obtained the funding to support this study. All authors have read and approved the final paper.

\section{Author details}

${ }^{1}$ State Key Laboratory of Oncology in South China, Collaborative Innovation Center for Cancer Medicine, Sun Yat-Sen University Cancer Center, Guangzhou 510060, China. ${ }^{2}$ Department of Basic Medicine, Guiyang College of Traditional Chinese Medicine, Guiyang 550002, China. ${ }^{3}$ Department of Breast Cancer, Sun Yat-Sen University Cancer Center, Guangzhou 510060, China. ${ }^{4}$ Department of Radiation Oncology, The First Affiliated Hospital of Guangzhou Medical University, Guangzhou 510060, China. ${ }^{5}$ Department of Breast Cancer, Sun YatSen University Cancer Center, Guangzhou 510060, China

\section{Conflict of interest}

The authors declare that they have no conflict of interest.

\section{Publisher's note}

Springer Nature remains neutral with regard to jurisdictional claims in published maps and institutional affiliations.

Supplementary Information accompanies this paper at (https://doi.org/ 10.1038/s41419-019-1705-z).

Received: 17 February 2019 Accepted: 23 May 2019

Published online: 17 June 2019

\section{References}

1. Ferlay, J. et al. Cancer incidence and mortality worldwide: sources, methods and major patterns in GLOBOCAN 2012. Int. J. Cancer 136, E359-E386 (2015).
2. Fidler, M. M. et al. Cancer incidence and mortality among young adults aged 20-39 years worldwide in 2012: a population-based study. Lancet Oncol. 18, 1579-1589 (2017).

3. DeSantis, C. E., Ma, J., Goding Sauer, A., Newman, L. A. \& Jemal, A. Breast cancer statistics, 2017, racial disparity in mortality by state. CA Cancer J. Clin. 67, 439-448 (2017)

4. Weigelt, B., Peterse, J. L. \& van't Veer, L. J. Breast cancer metastasis: markers and models. Nat. Rev. Cancer 5, 591-602 (2005).

5. Scully, O. J., Bay, B. H., Yip, G. \& Yu, Y. Breast cancer metastasis. Cancer Genom. Proteom. 9, 311-320 (2012).

6. Bartel, D. P. MicroRNAs: genomics, biogenesis, mechanism, and function. Cell 116, 281-297 (2004).

7. Xue, J. et al. MiRNA-621 sensitizes breast cancer to chemotherapy by suppressing FBXO11 and enhancing p53 activity. Oncogene 35, 448-458 (2016).

8. Niu, J. et al. Induction of miRNA-181a by genotoxic treatments promotes chemotherapeutic resistance and metastasis in breast cancer. Oncogene 35, 1302-1313 (2016).

9. Mulrane, L., McGee, S. F., Gallagher, W. M. \& O'Connor, D. P. miRNA dysregulation in breast cancer. Cancer Res. 73, 6554-6562 (2013).

10. Zare, M., Bastami, M., Solali, S. \& Alivand, M. R. Aberrant miRNA promoter methylation and EMT-involving miRNAs in breast cancer metastasis: Diagnosis and therapeutic implications. J. Cell Physiol. 233, 3729-3744 (2018).

11. Calin, G. A. \& Croce, C. M. MicroRNA signatures in human cancers. Nat. Rev Cancer 6, 857-866 (2006).

12. Moltzahn, F. et al. Microfluidic-based multiplex qRT-PCR identifies diagnostic and prognostic microRNA signatures in the sera of prostate cancer patients. Cancer Res. 71, 550-560 (2011).

13. Wong, N. et al. Prognostic microRNA signatures derived from The Cancer Genome Atlas for head and neck squamous cell carcinomas. Cancer Med. $\mathbf{5}$, 1619-1628 (2016).

14. Qu, Z. et al. Exosomal miR-665 as a novel minimally invasive biomarker for hepatocellular carcinoma diagnosis and prognosis. Oncotarget 8, 80666-80678 (2017).

15. Li, C. et al. Characterization and selective incorporation of small non-coding RNAs in non-small cell lung cancer extracellular vesicles. Cell Biosci. 8, 2 (2018).

16. Chen, J. et al. Screening of differential microRNA expression in gastric signet ring cell carcinoma and gastric adenocarcinoma and target gene prediction. Oncol. Rep. 33, 2963-2971 (2015)

17. Dong, C. et al. MicroRNA-665 suppressed the invasion and metastasis of osteosarcoma by directly inhibiting RAB23. Am. J. Transl. Res. 8, 4975-4981 (2016).

18. Zhou, B., Guo, W., Sun, C., Zhang, B. \& Zheng, F. Linc00462 promotes pancreatic cancer invasiveness through the miR-665/TGFBR1-TGFBR2/SMAD2/3 pathway. Cell Death Dis. 9, 706 (2018).

19. $\mathrm{Si}, \mathrm{H}$. et al. Circulating microRNA-92a and microRNA-21 as novel minimally invasive biomarkers for primary breast cancer. J. Cancer Res. Clin. Oncol. 139 223-229 (2013).

20. Liu, N. et al. Prognostic value of a microRNA signature in nasopharyngeal carcinoma: a microRNA expression analysis. Lancet Oncol. 13, 633-641 (2012).

21. Wei, R. et al. Clinical significance and prognostic value of microRNA expression signatures in hepatocellular carcinoma. Clin. Cancer Res. 19, 4780-4791 (2013).

22. Wang, H., Ach, R. A. \& Curry, B. Direct and sensitive miRNA profiling from lowinput total RNA. RNA 13, 151-159 (2007)

23. Kobayashi, $\mathrm{H}$. et al. Diagnosis of extraskeletal myxoid chondrosarcoma in the thigh using EWSR1-NR4A3 gene fusion: a case report. J. Med. Case Rep. 10, 321 (2016).

24. Huang, Y., Zhang, J., Cui, Z. M., Zhao, J. \& Zheng, Y. Expression of the CXCL12/ CXCR4 and CXCL16/CXCR6 axes in cervical intraepithelial neoplasia and cervical cancer. Chin. J. Cancer 32, 289-296 (2013).

25. Jolly, M. K. et al. Implications of the hybrid epithelial/mesenchymal phenotype in metastasis. Front. Oncol. 5, 155 (2015).

26. Rodriguez Calleja, L. et al. DeltaNp63alpha silences a miRNA program to aberrantly initiate a wound-healing program that promotes TGFbeta-induced metastasis. Cancer Res. 76, 3236-3251 (2016).

27. Wenzl, K., Troppan, K., Neumeister, P. \& Deutsch, A. J. The nuclear orphan receptor NR4A1 and NR4A3 as tumor suppressors in hematologic neoplasms. Curr. Drug Targets 16, 38-46 (2015).

28. Agaram, N. P., Zhang, L., Sung, Y. S., Singer, S. \& Antonescu, C. R. Extraskeletal myxoid chondrosarcoma with non-EWSR1-NR4A3 variant fusions correlate with rhabdoid phenotype and high-grade morphology. Hum. Pathol. 45, 1084-1091 (2014). 
29. Mullican, S. E. et al. Abrogation of nuclear receptors Nr4a3 and Nr4a1 leads to development of acute myeloid leukemia. Nat. Med. 13, 730-735 (2007).

30. Yeh, C. M. et al. Epigenetic silencing of the NR4A3 tumor suppressor, by aberrant JAK/STAT signaling, predicts prognosis in gastric cancer. Sci. Rep. $\mathbf{6}$, 31690 (2016).

31. Shimizu, R. et al. Possible role of intragenic DNA hypermethylation in gene silencing of the tumor suppressor gene NR4A3 in acute myeloid leukemia. Leuk. Res. 50, 85-94 (2016).

32. Busch, S. et al. TGF-beta receptor type-2 expression in cancer-associated fibroblasts regulates breast cancer cell growth and survival and is a prognostic marker in pre-menopausal breast cancer. Oncogene 34, 27-38 (2015).

33. Paiva, C. E. et al. Absence of TGF-betaRIl predicts bone and lung metastasis and is associated with poor prognosis in stage III breast tumors. Cancer Biomark. 11, 209-217 (2012).

34. Adeyinka, A. et al. Activated mitogen-activated protein kinase expression during human breast tumorigenesis and breast cancer progression. Clin. Cancer Res. 8, 1747-1753 (2002).

35. Bartholomeusz, C. et al. MEK inhibitor selumetinib (AZD6244; ARRY-142886) prevents lung metastasis in a triple-negative breast cancer xenograft model. Mol. Cancer Ther. 14, 2773-2781 (2015).

36. Calvayrac, O. et al. NOR-1 modulates the inflammatory response of vascular smooth muscle cells by preventing NFkappaB activation. J. Mol. Cell Cardiol. 80, 34-44 (2015).

37. Svoronos, A. A., Engelman, D. M. \& Slack, F. J. OncomiR or tumor suppressor? The duplicity of MicroRNAs in cancer. Cancer Res. 76, 3666-3670 (2016).
38. Bure, l. et al. The expression of hematopoietic progenitor cell antigen CD34 is regulated by DNA methylation in a site-dependent manner in gastrointestinal stromal tumours. Int. J. Cancer 141, 2296-2304 (2017).

39. Jin, X., Guan, Y., Sheng, H. \& Liu, Y. Crosstalk in competing endogenous RNA network reveals the complex molecular mechanism underlying lung cancer. Oncotarget 8, 91270-91280 (2017).

40. Liu, J. et al. MicroRNA665 suppresses the growth and migration of ovarian cancer cells by targeting HOXA10. Mol. Med. Rep. 18, 2661-2668 (2018).

41. Prashad, N. miR-665 targets c-MYC and HDAC8 to inhibit murine neuroblastoma cell growth. Oncotarget 9, 33186-33201 (2018).

42. $\mathrm{Hu}, \mathrm{Y}$. et al. miR-665 promotes hepatocellular carcinoma cell migration, invasion, and proliferation by decreasing Hippo signaling through targeting PTPRB. Cell Death Dis. 9, 954 (2018).

43. Slotta-Huspenina, J. et al. MicroRNA expression profiling for the prediction of resistance to neoadjuvant radiochemotherapy in squamous cell carcinoma of the esophagus. J. Transl. Med. 16, 109 (2018).

44. Marti-Pamies, I., Canes, L., Alonso, J., Rodriguez, C. \& Martinez-Gonzalez, J. The nuclear receptor NOR-1/NR4A3 regulates the multifunctional glycoprotein vitronectin in human vascular smooth muscle cells. FASEB J. 31, 4588-4599 (2017)

45. Nagaoka, M. et al. The orphan nuclear receptor NR4A3 is involved in the function of dendritic cells. J. Immunol. 199, 2958-2967 (2017).

46. Garattini, E. et al. Lipid-sensors, enigmatic-orphan and orphan nuclear receptors as therapeutic targets in breast-cancer. Oncotarget 7, 42661-42682 (2016). 